Journal of Optics A: Pure and Applied Optics, Vol. 8, No. 7, pp S430-S444

\title{
Fibre Optic Sensors with Nano-Structured Coatings
}

\author{
Stephen W. James and Ralph P. Tatam \\ Optical Sensors Group \\ Centre for Photonics and Optical Engineering \\ School of Engineering \\ Cranfield University \\ Cranfield MK43 0AL UK.
}

Tel 01234754623

Fax 01234752452

Emails.w.james@cranfield.ac.uk

Short title: Fibre Optic Sensors with Nano-Structured Coatings

PACS: 42.70.Jk, 42.79.Gn, 42.81.Pa, 42.81.Qb 


\section{Abstract}

A review of optical fibre devices employing nano-structured coatings is presented. Active and passive coatings, deposited using the Langmuir Blodgett and Electro-static Self Assembly techniques, have been utilised to influence the transmission spectrum of optical fibres, with the aim of developing sensor elements and in-line components for telecommunications systems. Coupled waveguide, fibre grating and miniature Fabry-Perot cavities are described. 


\section{Introduction}

The ability to interact with and/or control the properties of light propagating in optical fibres is of fundamental importance in both optical fibre sensor and optical telecommunication technologies. By interacting with the propagating modes it is possible to make sensors for a range of measurands and also to control the properties of the guided light, producing a family of functional devices such as tuneable filters and modulators.

The use of active and passive nano-structured coatings deposited onto optical fibres as a means for controlling the transmission spectrum of an optical fibre is reviewed within this paper. The use of thin film deposition techniques offering the ability to control the film thickness on the nanometer scale is significant, as this facilitates the optimisation of the interaction of the guided mode with the coating.

The devices created in this fashion offer an exciting prospect for the development of optical fibre sensors and devices. The coatings may be engineered to change their optical properties in response to an external stimulus. A number of configurations are discussed which facilitate interaction between modes of the optical fibre and the coatings, offering techniques for monitoring changes in the coating's optical properties. The sensors offer the advantages common to all fibre optic sensors: immunity to electromagnetic interference, small dimensions, light weight and the prospect for multiplexed arrays. 


\section{Nano-structured coating deposition techniques}

\subsection{Introduction}

The two major nano-coating techniques that will be discussed in this section are electrostatic selfassembly (ESA) and Langmuir-Blodgett (LB) deposition. Each facilitates the deposition of a surface coating one molecular layer at a time - giving nm scale thickness control. Both techniques allow the formation of thin films at room temperature and pressure.

\subsection{The Langmuir Blodgett Technique}

The LB technique allows the manipulation of material at the molecular level ${ }^{1,2}$. The technique is based on the fabrication of organic monolayer films, which are first oriented on a subphase and subsequently transferred, layer by layer, onto a solid surface at room temperature and molecule specific pressure. This offers high resolution control over the film thickness $(1 \mathrm{~nm}-3 \mathrm{~nm}$ per layer), and is thus ideal for waveguide applications.

Molecules constituting the LB film are usually amphiphilic, i.e. they have hydrophobic and hydrophilic tail or head groups ${ }^{1,2}$. Classic examples of these molecules are fatty acids such as stearic acid and $\omega$-tricosenoic acid. In the case of stearic acid, the carboxylic acid group acts as the hydrophilic portion, while the $\mathrm{C}_{17} \mathrm{H}_{35}$ hydrocarbon acts as a hydrophobic tail. A monolayer of the material is formed by applying the molecules to the surface of a subphase in the form of a solution. The solvent is allowed to evaporate, leaving the molecules dispersed across the whole of the water surface and oriented with the hydrophobic part upwards and the hydrophilic part in the water, producing a floating monolayer. Reducing the surface area by means of a moving barrier, the molecules begin to repel one another, modifying the surface pressure ${ }^{3}$. The change in surface pressure may be monitored to produce a surface pressure isotherm, such as that shown in figure 1 . The isotherm has three distinct phases; gas, liquid condensed and solid. 
At the onset of compression, the molecules are randomly oriented, and experience weak interaction with each other, behaviour analogous to a two dimensional gas. Upon further compression the molecules are pushed closer together as the hydrophobic tails of the molecules begin to lift from the surface. The molecules undergo a transition from the gas phase to a liquid-condensed phase, which is accompanied by a steep rise in surface pressure. Further compression reduces the surface area occupied by each molecule close to its cross-sectional area, producing a solid phase in which the molecules are densely packed and in which the molecules form an ordered array. From this phase, the molecules may be transferred to a solid substrate, forming an LB film ${ }^{1,2}$. LB films may consist of a single chemical species, this homogeneity not being available to the ESA method, which requires alternate layers of oppositely charged materials as will be discussed in section 2.3.

\subsubsection{Deposition of Langmuir-Blodgett Films}

An LB film can be deposited by passing a solid substrate such as a glass slide or optical fibre vertically through a condensed Langmuir layer. The surface pressure is monitored and kept constant during deposition by decreasing the surface area as the molecules are removed from the subphase and deposited on the substrate, ensuring that the solid phase is maintained. The vertical motion can be either down into the water (downstroke), or up, out of the water (upstroke). If the surface of the substrate is prepared such that it is hydrophilic, the first monolayer is transferred like a carpet as the substrate is raised through the Langmuir layer. The curve of the meniscus pushes the compressed monolayer onto the substrate. On the downstroke, the hydrophobic tail groups of the floating monolayer are attracted to those of the deposited LB film, thus depositing a second layer. Repeated dipping allows the film to be built one layer at a time, using these nanoscale building blocks. This represents the most common configuration, and is illustrated in figure 2 (a), with the adjacent layers packing head to head and tail to tail, and the overall film is termed Y-type or centrosymmetric ${ }^{4}$. 
A small number of materials can be deposited as either X- or Z-type, non centrosymmetric, LB films, offering the possibility of exploring non-linear optical processes such as second harmonic generation, and the electro-optic effect ${ }^{5,6,7}$. In these cases, layers are only deposited on the downstroke (X-type), or upstroke (Z-type) of the substrate, as illustrated in figure 2 (b).

\subsection{Electrostatic Self Assembly}

ESA is a technique for the deposition of multilayered films of magnetic ${ }^{8}$, electrically conductive ${ }^{9}$, and nonlinear optical materials ${ }^{10}$ onto a substrate. The basis of the technique is the alternate dipping of a charged substrate into solutions of cationic and anionic polymers. The growth of the film is based on electrostatic attraction between the opposite charges of molecules in alternate monolayers $^{11}$.

The ESA process is shown schematically in figure 3. A positively charged surface is immersed in a solution containing an anionic polyelectrolyte. A monolayer of the polyanion is adsorbed and the surface charge is reversed. The substrate is rinsed in pure water and is subsequently immersed in the solution containing the cationic polyelectrolyte. A second monolayer is adsorbed, and the original surface charge is restored. By repeating these steps, alternating multilayer films may be deposited ${ }^{11}$.

The LB and ESA techniques offer a number of different features. For ESA, multilayer formation is based on the attraction of opposite charges. This it is possible to incorporate more than two molecules into the multilayer, simply by immersing the substrate in as many solutions of polyeletrolytes, or appropriately charged inorganic nano-clusters, as desired, provided the charge reverses from layer to layer. Aperiodic multilayer assemblies may be prepared, offering more versatility than the Langmuir-Blodgett technique. Thus the individual layer composition and thickness can be controlled, and the properties of the film are determined by the polymers, the 
nano-particles and the long-range physical order of the layers. The immersion based process facilitates deposition on substrates of various sizes, shapes and materials, including metals, plastics, ceramics and semiconductors ${ }^{12}$.

\section{COUPLED WAVEGUIDE DEVICES}

\subsection{Introduction}

Fibre optic components based upon coupled waveguides have been studied extensively, and many standard fibre optic components, for example, directional couplers, are based upon this principle. The principle of operation concerns the transfer of energy between two dielectric waveguides, which occurs when the waveguides are in close proximity to one another, such that the evanescent fields of the propagating modes can interact when the waveguides are phase matched.

A thin film overlay deposited onto a substrate that is an optical waveguide may itself act as a waveguide, provided its index is higher than that of the substrate. The phase matching condition is dependent upon parameters including the dimensions and refractive indices of the two waveguides and is given by

$$
\frac{2 \pi}{\lambda_{m}} d \sqrt{n_{0}^{2}-n_{e f f}^{2}}=m \pi+\Phi_{a}+\Phi_{C o}
$$

Where $n_{\text {eff }}$ is the effective index of the mode propagating in the substrate, $d$ is the thickness of the overlay, $\mathrm{n}_{0}$ is the refractive index of the overlay, $\Phi_{\mathrm{a}}$ and $\Phi_{\mathrm{co}}$ are polarisation dependent phase change terms occurring at the air and substrate boundaries and $\lambda_{\mathrm{m}}$ is the synchronous wavelength for coupling to the $\mathrm{m}^{\text {th }}$ order mode of the overlay waveguide. In the presence of strong differential waveguide dispersion between the guides, this produces a series of sharp channel dropping responses in the transmission spectrum (discussed further in section 3.3). The coupling wavelength 
is particularly sensitive to changes in the overlay thickness and so very accurate thickness control, as provided by the LB and ESA techniques, is required.

In the case of the devices to be discussed here, the coupled waveguides are formed by the core of the optical fibre and an overlay waveguide deposited on the optical fibre by the LB technique. Access to the evanescent field of the propagating mode of a single mode optical fibre requires removal of much of the cladding. This is generally achieved by tapering the optical fibre ${ }^{13}$, or by polishing the fibre ${ }^{14}$.

\subsection{Polished Fibre Devices}

Two polishing configurations are illustrated in figures 4 (a) and (b). In figure 4 (a) the optical fibre is first stripped of its polymer buffer jacket over a length of $15 \mathrm{~mm}$ and is subsequently fixed into a groove with a $250 \mathrm{~mm}$ radius of curvature cut into a glass block. Material is then removed from the top surface of the block using a rotary polishing machine. The technique is used in the fabrication of fibre components such as variable split ratio directional couplers ${ }^{14}$ and in fibre polarisers ${ }^{15}$. This technique has limitations for LB deposition, as the fibre interaction region occupies only a small fraction of the total surface area, and thus the majority of the film does not contribute to the interaction. The typical interaction length between the core and the overlay waveguide achieved using this technique is limited to $1-2 \mathrm{~mm}$. In addition, the differential polishing rates of the glass substrate, the silica fibre and the adhesives used to bond the fibre into the groove can also compromise the film deposition ${ }^{16}$.

An alternative method, the annular polishing technique ${ }^{17}$, is illustrated in figure 4 (b). The fibre is tensioned across a $25 \mathrm{~mm}$ diameter abrasive wheel. The polished fibre profile can be seen to afford a longer interaction length, of $5 \mathrm{~mm}-10 \mathrm{~mm}$, defined by the diameter of the wheel and the configuration of the tensioned fibre. 
Monitoring the polishing depth is critical to obtaining optimum performance of the polished fibre device. The oil drop method ${ }^{18,19}$ is commonly used, where a drop of refractive index matching oil is placed in the polished region, and its influence on the transmission of the fibre measured. If the refractive index of the oil matches the index of the propagating core mode, the electric field propagating in the overlay formed by the oil changes its characteristic from evanescent to radiative, with a concomitant decrease in the transmitted power. The decrease in transmission is strongly influenced by the thickness of the remaining cladding. From the transmission measurement it is possible to determine the amount to the evanescent field that has been accessed through the polishing process, and to determine the coupling strength.

An alternative technique involves operating the optical fibre below its cut off wavelength, such that it guides a small number of modes. As the polishing depth increases, these modes will be cut off in turn, leading to a series of step reductions in the transmitted power ${ }^{20}$.

The polished block approach and LB film deposition have been used to form a fibre optic polariser and modulator ${ }^{21}$. In this case, an LB film of Cadmium Stearate (molecular length $2.5 \mathrm{~nm}$ ) was deposited on a gold-coated single mode side polished optical fibre. The thickness of the LB film influenced the properties of the surface plasmon resonance based polariser, allowing optimisation of the extinction ratio for a film of thickness $500 \mathrm{~nm}$. Application of de electric fields of upto $50 \mathrm{MV} / \mathrm{m}$ across an LB film consisting of 41 layers of hemicyanine deposited onto a gold surface, upon which a gold film was sputtered to form the top electrode, allowed limited tuning of the extinction ratio $^{21,22}$.

\section{Channel dropping filters}


An example of a channel dropping filter is shown in figure 5, where an LB film of $\omega$-tricosenoic acid was deposited onto a single mode optical fibre polished using the rotary method ${ }^{23,24}$. Each transmission spectrum was obtained for a different number of LB layers deposited into the fibre. The graph shows that an increase in the film thickness by a molecular bi-layer $(\sim 5.8 \mathrm{~nm})$ results in a change in the coupling wavelength of $\sim 14 \mathrm{~nm}$. This illustrates the requirement for the use of a film deposition technique with a nano-scale thickness resolution for the fabrication of such components, to ensure operation at a specified wavelength.

A second consequence of the high sensitivity of the coupling wavelength upon the overlay's thickness is a concomitant dependence upon the refractive index of the material. Thus if the material changes its optical properties in response to an external stimulus, for example, exposure to a particular chemical species, or an applied voltage, this will also result in a shift in the synchronous wavelength, forming the basis of a sensor.

Figure 6 illustrates the sensitivity of the channel dropping response to temperature. This sensitivity arises from the differential thermo-optic coefficients, and thus temperature induced refractive index changes, of the optical fibre and the overlay waveguide ${ }^{24}$.

The ability of the LB film to act as a waveguide was used to capture fluorescence from a cyaninedye-impregnated film of cadmium arachidate deposited on the core of a multimode optical fibre that formed one arm of a $3 \mathrm{~dB}$ coupler. The fluorescence was re-coupled into the fibre, and monitored in reflection, as shown in figure $7^{25}$.

$$
\text { . }
$$

\subsection{Chemical sensor}

Deleted: 9

Deleted: …_...Page Break

A pH sensor was demonstrated by depositing LB film-forming materials known to be sensitive to $\mathrm{pH}$, taken from the family of merocyanine dyes, onto a side polished optical fibre. Merocyanine 
dyes contain a terminal oxygen atom that can be reversibly protonated, leading to a change in the absorption spectrum and thus a change in its refractive index. Two dyes were investigated. The absorption spectrum of 20 molecular layers of the first dye, E-N-docosyl-4-[2-(4-oxyphenyl) ethenyl] pyridinium inner salt (structure shown in figure 8(a)), deposited onto a glass slide, is shown in figure $8(\mathrm{~b})$ before and after exposure to $\mathrm{HCl}$ vapour $^{26,27}$. A channel dropping filter with a coupling wavelength of $725 \mathrm{~nm}$ was created by depositing 56 layers of the dye on an annularly polished fibre (cut-off wavelength of $630 \mathrm{~nm}$ ). A schematic of the device is shown in figure 9 . The response of the transmission spectrum of the device to exposure to solutions of varying $\mathrm{pH}$ yielded is shown in figure 10 (a), yeilding a linear response with a sensitivity of $18.8 \pm 0.8 \mathrm{~nm} / \mathrm{pH}$ and a resolution of $0.1 \mathrm{pH}$, as shown in figure 10 (b). The organic materials that may be deposited using the LB technique may be tailored to satisfy specific purposes. A second dye was investigated that has its absorption at longer wavelengths. The use of this dye would result in larger refractive index in the near infrared region of the spectrum, increasing sensitivity. This dye differed from the original by the addition of bromine atoms and replacing one carbon atom on the ethylene bridge with a nitrogen atom, Figure 11 (a). The response of the absorption spectrum to exposure to $\mathrm{HCl}$ vapour is shown in Figure $11(b)^{27} .178$ layers of the 2nd dye were deposited on an annularly polished fibre. The response to $\mathrm{pH}$ is shown in figure 12 (a). In this case, the wavelength shift of the central wavelength of the channel dropping response was nonlinear, as shown in figure 12 (b). A Kramers-Kronig model, in which experimentally determined transmission spectra of 50 layers of the dye deposited onto a glass slide recorded on exposure to solutions of differing $\mathrm{pH}$ were used to determine changes in the material dispersion of the overlay waveguide, was found to match well with the experimental results, as indicated by the solid line in figure $12(b)^{27}$.

\subsection{Optically non-linear nano-structured coatings}

As discussed in section 3.1, LB films may be deposited with a non-centrosymmetric structure, offering the possibility of exploiting non-linear optical processes, such as the electro-optic effect. 
This is particularly appealing, as LB film forming materials have been shown to have high nonlinear coefficients, with Pockels' coefficients of $100 \mathrm{pm} / \mathrm{V}$ being reported ${ }^{28}$. These materials may be deposited as Z-type LB films onto both glass slide and side polished single mode optical fibres, and characterised as non-linear materials by investigating second harmonic generation.

The first demonstration of SHG from a LB film deposited on an optical fibre was reported in [29]. A film of a hemicyanine dye was deposited in a Z-type configuration onto the core of an etched 600 $\mu \mathrm{m}$ diameter multi-mode optical fibre. A conversion efficiency of $10^{-6}$ was achieved for 80 layers of the dye when illuminated using a pulsed Nd:YAG laser source coupled into the fibre. The SHG signal was guided in the film, and the low value was attributed to the likely phase mismatch of the fundamental and SHG, and to the low energy density offered by the use of multimode fibre ${ }^{\text {Error! }}$ Bookmark not defined.

To improve the efficiency of coupling of light to the film, an optically nonlinear dye, E-Noctadecyl-4-[2-(4-dibutylaminophenyl) ethenyl]quinolinium octadecylsulfate ${ }^{30}$, which inherently forms Z-type structures and has a high nonlinear coefficient, was deposited on a side polished single mode optical fibre ${ }^{31}$. The channel dropping response of the coupled waveguide structure was monitored during deposition of the LB film until it was centred at $1064 \mathrm{~nm}$, the wavelength of the laser used as the pump for the SHG process. The resultant channel dropping response is shown in figure 13. For Z-type, non-centrosymmetric deposition, it is necessary for the monolayer to be transferred to the surface of the polished section of the fibre on the upstroke only. To achieve this in a consistent and reproducible fashion, a mechanism was developed to isolate the fibre from the floating Langmuir monolayer as it traverses the air-water interface ${ }^{32}$, using a cylindrical stainless steel tube that temporarily enclosed the fibre on the downstroke. 
Cerenkov radiation-type phase matching was exploited as this imposes the least stringent control of the parameters for frequency conversion ${ }^{33}$. In this regime, the fundamental beam is guided in the LB film and the generated second-harmonic is radiated into the substrate, as shown in figure 14.

Light from a Q-switched Nd: YAG laser, operating at $1064 \mathrm{~nm}$ with a pulse duration of $10 \mathrm{~ns}$ was coupled into the optical fibre. The second-harmonic intensity relative to the signal from a Y-cut quartz reference was monitored via photomultiplier tubes. The energy of the guided fundamental pump beam exiting the fibre was monitored continuously.

The region of fibre coated with the LB film was mounted on a Peltier thermoelectric element to allow thermal control of the resonant wavelength, $\lambda_{\mathrm{m}}$, and hence the amount of light coupled into the overlay. The second-harmonic intensity from the LB overlay is plotted as a function of fibre transmission in figure 15. Increased transmission is indicative of reduced coupling into the overlay and the second-harmonic intensity decreases as the square of the transmitted fundamental intensity. Varying the optical power coupled into the fibre, and therefore to the overlay, shown in figure 16. The slope is positive and, as expected from theory, the second-harmonic intensity increases as the square of the fundamental intensity.

\section{OPTICAL FIBRE LONG PERIOD GRATINGS}

\subsection{Introduction}

The devices discussed in the previous section relied upon the removal of a portion of the fibre cladding to allow the properties of the overlay material to influence the transmission characteristics of the optical fibre. Fibre optic long period gratings (LPGs) offer a method for facilitating the interaction of modes of the fibre with the LB coating with no requirement for removal of the cladding. LPGs are a relatively new class of fibre optic device that offer exciting properties for optical sensing applications ${ }^{34}$ and have already found application as gain flattening filters for 
erbium amplifiers. An LPG is an UV induced periodic modulation of the refractive index of the core of an optical fibre, with a period in the range $100 \mu \mathrm{m}-1000 \mu \mathrm{m}$. The small grating wavevector of an LPG promotes the coupling of light from the guided modes of the fibre to cladding modes, with the result that the transmission spectrum of a fibre containing an LPG consists of a number of attenuation bands centred at discrete wavelengths ${ }^{34}$.

The transmission spectrum of an LPG is shown in figure $17^{35}$, exhibiting the characteristic attenuation bands. The exact form of the spectrum and the centre wavelengths of the attenuation bands are sensitive to the local environment: temperature, strain, bend radius and refractive index of the medium surrounding the fibre ${ }^{36}$. The sensitivity to a particular measurand is dependent upon the period of the LPG and the order of the cladding mode to which the guided optical power is coupled, and thus is different for each attenuation band. This range of responses makes them particularly attractive for sensor applications, with the prospect for multi-parameter sensing using a single sensor element.

LPGs have a number of attractive properties that may be exploited for sensing applications. They exhibit sensitivity to the refractive index of the medium surrounding the fibre, which has been exploited to form sensors capable of measuring the refractive index of a solution, allowing concentrations of sodium chloride and ethylene glycol to be determined ${ }^{37}$. Such sensors are not chemical species specific, and are limited to operation with solutions with refractive indices less than or equal to the refractive index of the fiber cladding. Of more interest is the potential to deposit overlay materials that exhibit changes in their refractive index in response to their local environment. In this way, the LPG could form, for example, a tuneable loss filter $^{38}$, a temperature insensitive filter ${ }^{39}$ or a species-specific chemical sensor ${ }^{40}$.

\subsection{Deposition of Nanostructured Coatings onto Fibre Optic Long Period Gratings}


The central wavelengths and the minimum transmission of the LPG attenuation bands can exhibit a dependence on both the thickness and refractive index of an overlay material, even when the overlay has a refractive index higher than that of the cladding ${ }^{41}$.

The spectral response of an LPG of length $40 \mathrm{~mm}$, period $400 \mu \mathrm{m}$, to submersion in external media of different refractive indices is shown in figure 18, were the refractive index induced wavelength shift of the central wavelength of an attenuation band in the spectrum is plotted. The figure shows that the response to refractive index occurs over a range of approximately 1.400 to 1.456 . The upper limit is imposed by the refractive index of the cladding material. For refractive indices above this limit there is no wavelength response.

An LB film of $\omega$ tricosenoic acid, which has refractive index of 1.57 and a molecular length of 2.6 $\mathrm{nm}^{42}$, was deposited onto the optical fibre containing the LPG. A transmission spectrum was recorded after each bi-layer had been deposited.

The wavelengths of the $4^{\text {th }}, 5^{\text {th }}$ and $6^{\text {th }}$ attenuation bands are plotted as a function of overlay thickness in figure 19 (a). Figure 19 (b) shows the shift in the central wavelengths of the attenuation bands corresponding to coupling to the $5^{\text {th }}$ and $6^{\text {th }}$ cladding modes plotted as a function of the thickness of the film. The graph contains three distinct regions characterized by differences in the form and response of the transmission spectrum. The graph shows that for an overlay thickness of less than $250 \mathrm{~nm}$, (region A), the attenuation band suffers a wavelength shift of $\sim 5 \mathrm{~nm}$. For an overlay thickness of between $250 \mathrm{~nm}$ and $380 \mathrm{~nm}$, (region B), the amplitude of the attenuation bands in the transmission spectrum reduced to zero and thus no coupling wavelengths could be recorded. For an overlay thickness of over $380 \mathrm{~nm}$, (region C), the attenuation bands reappeared, but at a wavelength higher than originally observed in the absence of the overlay material. As the 
film thickness was increased further, the central wavelengths of the attenuation band returned towards their original value.

Similar effects have been observed for thin films deposited via the ESA technique ${ }^{43,44}$. Larger wavelength shifts, of upto $40 \mathrm{~nm}$, were observed for films of thickness $25 \mathrm{~nm}$, formed using Poly(allylamine hydrochloride as the polycation, and poly\{1-[4-(3-carboxy-4-hydroxy-phenylazo) benzenesulfonamido]-1,2- ethanediyl, sodium salt $\}$ was used as polyanion ${ }^{43}$. The larger wavelength shifts may be attributed to operating at longer wavelengths, and with the use of films of higher refractive index. It was also shown that careful control of the properties of the anion and cation allows control over the thickness and refractive index of the film and thus of the wavelength of the attenuation bands ${ }^{43}$.

The dependence of the central wavelengths of the attenuation bands upon the thickness and refractive index of the overlay has been modelled ${ }^{41}$. The effective refractive indices of the cladding modes were calculated as a function of wavelength and overlay thickness by considering the cladding-overlay system as a stack of thin films and employing the transfer matrix method ${ }^{45}$. The effective refractive index of the propagating core mode was calculated using the approach of Gloge $e^{46}$. Using the calculated dispersion of the core and cladding modes, the dependence of the central wavelengths of the LPG attenuation bands upon overlay thickness could be predicted. The model makes a number of approximations, in particular that the system may be modeled as a multilayer planar waveguide, and ignores the influence of the coupling efficiency between core and cladding modes upon the coupling wavelength and the depth of the attenuation bands. The model does however qualitatively predict the experimentally observed behaviour; however, this approach suffers from a number of limitations which result from the approximations made. The theoretical prediction of the variation of wavelength as a function of overlay thickness is illustrated in figure 
$20(\mathrm{a})^{41}$. As for the experimental results, if the order of the attenuation band is ignored, the wavelength shifts appear to be continuous as observed experimentally in figure 19 (a).

More rigorous theoretical treatments have provided similar predictions $\frac{43,47,48,49}{4 h e r e ~ t h e ~ L P G ~ i s ~}$ modeled using coupled mode theory, and in reference 50, where a step index waveguide geometry is modeled. Using a linearly polarised (LP), weakly guiding approximation, it has been shown that, as thickness of an overlay of refractive index higher than that of the cladding is increased, the overlay, itself a waveguide, become capable of guiding one of the cladding modes. During the transition to guidance, the effective index of the cladding mode varies rapidly, as evidenced by the rapid change in wavelength of the attenuation bands. In the absence of losses, i.e. an imaginary part of the refractive index, in the overlay it is the cladding mode with highest effective index (i.e. lowest order) that is guided. The transition is also characterized by a reorganization of the cladding modes in region B of figure 19 (b). The effective indices of the cladding modes are then increased to that of their nearest lower order mode in the absence of the overlay, as illustrated in figure 21. Further increases in overlay thickness cause this process to repeat $\frac{43}{\Delta}$

The LP approximation presented does not predict the disappearance of the attenuation bands unless the overlay exhibits a complex refractive index. In this case the attenuation of the cladding modes is predicted to increase rapidly in the transition region, which reduces the efficiency of coupling between core and cladding modes, and, as a result, reduces the extinction of the attenuation bands $\frac{47}{\text { }}$ In this case, it is not necessarily the lowest order cladding mode that is guided, and the modal reorganization is more complex. Attenuation bands corresponding to coupling to higher order cladding modes than that which is guided by the overlay shift to the immediate lower order cladding-mode resonance condition. Attenuation bands corresponding to coupling to cladding modes of order lower than that which is guided initially suffer a blue shift before recovering their original resonance wavelength. Extension to consider the hybrid modes shows that, within one LP 
is observed.

The simplistic model discussed previously can be used to predict the response of the attenuation bands' central wavelengths to deposition of films of differing index, as illustrated in figure 22 . It can be seen that, as the index of the overlay material increases, the region of high sensitivity moves to lower thicknesses. It is possible to predict the significant sensitivity of the attenuation bands to changes in the refractive index of the overlay, assuming a constant overlay thickness, as shown in figure 23. This indicates the potential for developing sensors based upon nm scale coatings that change their refractive index in response to an external stimulus.

The use of LPGs as refractive index sensors, to determine the concentration of aqueous solutions is well established ${ }^{37}$. The model described above predicts that the sensitivity of an LPG refractometer can be enhanced by the deposition of a nanometer scale overlay, with the degree of enhancement dependent upon the refractive index and thickness of the overlay. This has recently been demonstrated experimentally, using films deposited by the LB and ESA techniques ${ }^{52,53}$.

The wavelength shifts offer many new prospects for optical fiber sensor configurations. Of particular interest is the transition from region $\mathrm{A}$ to region $\mathrm{C}$, since this introduces a large wavelength shift for a small change in the optical thickness of the overlay. If a film of appropriate thickness is deposited, then a small refractive index change could cause a large wavelength shift, offering the prospect of developing a highly sensitive sensor, or an optical modulator. This has been exploited by dip-coating the nanoporous crystalline $\delta$ from of Syndiotactic Polystyrene (sPS) onto an LPG. sPS can reversibly adsorb analytes of suitable size and shape, with a concomitant change in refractive index. The sensor was shown to be capable of sub-ppm detection of chloroform in 
Concatenated or in-series LPGs allow the production of finer detail within the transmission spectrum $^{55}$. The concatenated LPGs act as a Mach-Zehnder interferometer, as illustrated in figure 24. Light coupled into the cladding by the first LPG is re-coupled into the core by the second LPG, where it interferes with the light that propagated in the core, producing interference fringes within the attenuation bands, as illustrated in figure 25 . The deposition of a nano-structured film onto concatenated LPGs has been reported ${ }^{56}$. Films deposited on the length of optical fibre separating the LPGs affect only the interference fringes, while films deposited along the entire device influence both the fringes and the attenuation band envelope. Figure 26 shows the response of the transmission spectrum to the LB deposition of a film of $\omega$-tricosenoic acid onto a $20 \mathrm{~mm}$ length of fibre separating two identical LPGs of period $400 \mu \mathrm{m}$ and length $5 \mathrm{~mm}$. The initial blue shift of the fringes is followed by a reduction in visibility, corresponding to region B of figure 19 (b), and by a subsequent reappearance of the fringes $\frac{56}{x}$

\section{Sub-micron Fabry-Perot interferometers formed using nanostructured coatings}

Sub-micron Fabry-Perot interferometers formed at the end of optical fibres have been used as low-

Inserted: ${ }^{55}$

Deleted: - Page Break

Deleted: ๆ mass low-cost one time sensors for gases such as ozone on Mars ${ }^{57}$, and elsewhere as sensor elements for a range of measurands including temperature, vibration and pressure ${ }^{58}$. Several methods have been reported for the fabrication of an optical cavity on the end of an optical fibre such as vapour deposition of metals ${ }^{59}$. A constraint of the all-metallic sensing elements is their inherent limitation compared with the sensing capabilities of organic materials. Also, in general it is difficult to obtain high precision in the film thickness for short cavities $(<2 \mu \mathrm{m})$. These limitations have been overcome using the $\mathrm{LB}^{60}$ and $\mathrm{ESA}^{61}$ deposition techniques, demonstrating thickness control of $c a .3-5 \mathrm{~nm}$ layer $^{-1}$ to a total film thickness of $1 \mu \mathrm{m}$ in the formation of Fabry-Perot type 
interferometric optical cavities on the end of the optical fibre in which the optical fiber/ film interface formed the first mirror, and the film/air interface formed the second mirror, as shown in figure 27.

To facilitate the fabrication of a Fabry-Perot type interferometric optical cavity using the LB technique, the LB trough dipper mechanism was modified to allow the film to be deposited on the cleaved end face of the optical fibre. For the ESA technique, the cavity was formed by alternately dipping the end of the fibre in a solution of poly(sodium 4-styrene-sulfonate) salt and a solution of gold colloids in polydiallyldimethyl-ammonium chloride, washing in pure water between ${ }^{61}$ x

In general, if the molecular length is $l$ and the number of bilayers is $\mathrm{m}$, then the cavity length is $\mathrm{d}=$ $2 \mathrm{~m} l \cos \varphi$ where $\varphi$ is the tilt angle of the molecules when deposited onto the substrate. The optical phase change experienced by light undergoing a double pass through the cavity is

$\varphi=\frac{4 \pi \mathrm{n}_{2} \mathrm{~d}}{\lambda}$

where $\varphi$ is the free space wavelength of the source and $n_{2}$ is the refractive index of the cavity.

The fabricated cavity has low reflectance at $\mathrm{M}_{1}$, due to the small difference in refractive index between the fibre and the cavity material, and thus acts similarly to a two beam interferometer, where the transfer function will be cosinusoidal, of the form

$\mathrm{I} \approx \mathrm{I}_{0}(1+\mathrm{V} \cos \varphi)$

where $\mathrm{V}$ is the visibility. 
In the case of LB deposited cavities, the cavity material, $\tilde{\omega}$ tricosenoic acid, was transferred on to the end of the fiber by vertical deposition, with the fibre's cleaved face orthogonal to the plane of the film, using a modified dipper mechanism. Y-type structures, in which the amphiphilic molecules pack head-to-head and tail-to-tail, were obtained by multiple passes through the film.

The experimental layout shown in figure 28 was used to monitor the formation of the cavity, the light source being a super-luminescent diode with a central wavelength of $830 \mathrm{~nm}$ and a $3 \mathrm{~dB}$ bandwidth of $30 \mathrm{~nm}$. This was coupled to the cavity via a $3 \mathrm{~dB}$ directional coupler using single mode optical fibre with cut-off wavelength $730 \mathrm{~nm}$. As the cavities formed were of the order of 1 $\mu \mathrm{m}$ in length, a broad band source with a coherence length of $15 \mu \mathrm{m}$ was sufficient to monitor their fabrication. The optical power of the source and the reflected signal from the cavity were monitored on separate matched photo-detectors. Following the deposition of each bilayer, at an incremental thickness of $c a .3 \mathrm{~nm}$, the ratio of the reflected to incident intensity was recorded. This ratio is plotted versus number of bilayers in figure 29 . The reflected signal varies periodically with the cavity thickness, showing the interferometric nature of the cavity.

As reported for cavities formed using $\operatorname{ESA}^{61}$, figure 29 shows some attenuation with cavity thickness as a result of absorption and scattering within the film. There is some asymmetry, possibly resulting from incomplete deposition of the multilayer. The low visibility of the fringes is a result of the small difference in refractive index difference between the LB/fibre and LB/ air interfaces, and also due to scattering loses in the cavity. Higher visibility has been demonstrated using the ESA technique, using films of higher refractive index.

Knowledge of the dispersion of the material would allow the cavity to be monitored at different wavelengths and thus facilitate signal processing of the cavity when deployed as a sensing element. 
The facility to control the optical thickness of the cavity on a nanometer scale permits the formation of high spatial resolution optical fiber sensors and the potential for sensors with fast response times. In addition, the film-forming materials may be designed to change their optical characteristics when reacting to a particular chemical species, or, in the case of LB films, form non-centrosymmetric films that exhibit non-linear optical properties, opening up the potential for high spatial resolution sensing based on non-linear optics.

A sub-mcron cavity fabricated via the ESA technique was used to demonstrate a humidity sensor. A solution of poly (sodium 4-styrene-sulfonate) salt (PSS) formed the anionic electrolyte. A solution of gold colloids protected by a polymer coating, poly (diallyldimethyl ammonium chloride) (PDDA), was used to form the cationic electrolyte (Au:PDDA). A bi-layer structure of thickness $309 \mathrm{~nm}$ with the form [Au:PDDA ${ }^{+} / \mathrm{PSS}^{-}$] was deposited on the endface of an optical fibre. The operation of the sensor was not based upon the interferometric nature of the cavity. Rather, the hygroscopic property of the coating material was exploited. The trapping of water drops at the surface of the coating, the size of which is dependent on the RH, resulted in changes in the reflectivity of the end mirror of the cavity ${ }^{12,62}$. The sensor showed reversible operation over the relative humidity range $11.3 \%$ to $100 \%$, with a response time of $1.5 \mathrm{~s}$, making it suitable for monitoring human respiration. The affinity of dichloromethane to a polymer coating composed of poly (diallyldimethyl ammonium chloride) (PDDA) and the dye Poly S-119 has been used in a similar way to form a dichloromethane sensor. Here the change in the reflected optical power was achieved by the capture of drops of dichloromethane on the coating ${ }^{63}$. LB films have been employed in similar configurations. The reflection from a lutetium bisphthalocyanine film deposited onto the end of an optical fibre was monitored when exposed to acetic acid, ethanol, $n$ butyl acetate and hexanal ${ }^{64}$. ESA has been used to demonstrate an ammonia sensor based upon thin films of zirconia ( $\mathrm{ZrO} 2)$ nanoclusters and poly(sodium-4-styrenesulfonate) salt deposited on the 
cleaved ends of optical fibres ${ }^{65}$. The sensor was unaffected by humidity and the presence of volatile organic compounds.

A hydrogen peroxide $\mathrm{H}_{2} \mathrm{O}_{2}$ sensor was formed by mixing an indicator dye, Prussian blue, in the polycation layer. The operation of the sensor relied upon measurement of the rate of change of the reflected intensity, which was related to the change in the absorbance of the indicator caused by the diffusion of the $\mathrm{H}_{2} \mathrm{O}_{2}$ into the nanostructure. A cavity of thickness 17 bilayers were found to provide the optimum sensitivity, and a capping layer was used to prevent loss of the indicator dye $e^{66,67}$.

The ability of ESA to form multilayer coatings using more than two different materials has allowed the fabrication of filters formed from quarter-wavelength stacks on the end faces of cleaved and polished optical fibres ${ }^{68}$. Such structures formed on the end of multi-mode fibre have been used to bias the operating wavelength of the sensor, and to thus allow the possibility of wavelength division multiplexing of sensor arrays $\frac{68}{x}$

\section{SUMMARY}

The properties of fibre optic devices employing nano-structured coatings have been reviewed. The deposition of active and passive coatings using the Langmuir Blodgett and Electro-static Self Assembly techniques, have been described. Their use to form coupled waveguides and miniature Fabry-Perot cavities, and their use to influence the transmission spectra of fibre optic long period gratings has been detailed. The ability to manipulate material deposition onto optical fibres at the nanoscale has lead to a new class of passive and active photonic devices with exciting potential in sensing and signal processing for future sensor and communication systems. 


\section{ACKNOWLEDGEMENTS}

The authors would like to acknowledge the Engineering and Physical Sciences Research Council, EPSRC, UK and the Royal Society for their support. 


\section{REFERENCES}

1 I.R Peterson, Langmuir-Blodgett films, J.Phys. D: Appl.Phys. 23, 379-395 (1990)

2 M.C. Petty Langmuir-Blodgett films, Cambridge University Press, 1996.

3 I.R Peterson, Langmuir-Blodgett films, in G.J. Ashwell (ed) Molecular Electronics, Research Studies Press, Taunton, UK, 1991.

R.A. Hann, Molecules for Langmuir Blodgett film formation, Philosophical Trans.Roy. Soc. A330, 141-152 (1990).

5 G.J. Ashwell, P.D. Jackson, W.A. Crossland, Non-centrosymmetry and second harmonic generation in Z-type Langmuir Blodgett films, Nature, 368, 438-440 (1994).

6 G.J. Ashwell, P.D. Jackson, G. Jeffries, I.R. Gentle and C.H.L. Kennard, Controlling the structures of transparent Langmuir-Blodgett films for nonlinear-optical applications, J.Mat.Chem. 6, 137-141 (1996).

7 L.M. Hayden, S.T. Kowel and M.P. Srinivasan, Enhanced second harmonic generation from multilayerd Langmuir Blodgett films of dye, Opt.Comm. 61, 351-356 (1987)

8 Y. Liu, Y. Wang and R.O. Claus, Layer-by-layer electrostatic self-assembly of nanoscale $\mathrm{Fe}^{3} \mathrm{O}^{4}$ particles and polyimide precursor on silicon and silica surfaces, Appl. Phys. Lett. 71, 2265-2267, (1997).

9 Y. Liu, Y. Wanga and R. O. Claus Layer-by-layer ionic self-assembly of Au colloids into multilayer thin-films with bulk metal conductivity Chem. Phys. Lett. 298, 315-319 (1998).

10 K.M. Lenahan, Y.-X. Wang, Y. Liu, R.O. Claus, J.R. Heflin, D. Marciu and C. Figura, Novel polymer dyes for nonlinear optical applications using ionic self-assembled monolayer technology Adv. Mater. 10, 853-855, 1998.

11 G. Decher, J. D. Hong and J. Schmitt, Buildup of ultrathin multilayer films by a self-assembly process: III. Consecutively alternating adsorption of anionic and cationic polyelectrolytes on charged surfaces, Thin Solid Films 210-211, 831-835 (1992). 
12 F. J. Arregui, Y. Liub, I. R. Matiasa and R. O. Claus Optical fibre humidity sensor using a nano Fabry-Perot cavity formed by the ionic self-assembly method, Sensors and Actuators B: Chemical 59, 54-59 (1999).

13 H.S. MacKenzie and F.P. Payne, Evanescent field amplification in a tapered single mode fibre, Electron.Lett. 26, 130-132 (1990).

14 R.A. Bergh, G. Kotler and H.J. Shaw, Single mode fibre optic directional coupler, Electron.Lett. 16, 260-261 (1980).

15 K.Y. Hsu, S.P. Ma, K.F. Chen, S.M. Tseng, J.I. Chen Surface-polariton fiber polarizer: Design and experiment Japanese J.App. Phys. Part 2-Letters 36 L488-L490 (1997)

16 R B Charters, S E Staines and R P Tatam Passive and Active In-Line Fibre Components using Langmuir-Blodgett films on Monomode Optical Fibre, in Fiber Optic Materials and Components. in Proc SPIE. 2290 , pp 304-314, (1994) .

17 C.D. Hussey and J.D. Minelly, Optical fibre polishing with a motor-driven polishing wheel, Electron.Lett. 24, 805-807 (1988).

18 O.G. Leminger and R. Zengerle, Determination of single mode fibre coupler design parameters from loss measurements, IEEE J.Lightwave Technol. LT-3B, 864-867 (1985)

19 M.J.F. Digonnet, J.R. Feth, L.F. Stokes and H.J. Shaw, Measurement of the core proximity in polished fibre substrates and couplers, Opt.Lett. 10, 463-465 (1985).

20 D. Flannery, Fibre optic chemical sensing using Langmuir-Blodgett overlay waveguides, PhD Thesis, Cranfield University (1998).

21 R Zhu, Y Wei, B Scholl, H.J. Schmitt, In-line optical-fiber polarizer and modulator coated with langmuir-blodgett-films, IEEE Photon .Technol. Lett. 7, 884-886 (1995)

22 R Zhu, Y Wei, B Scholl, H.J. Schmitt, Modulation of the polarization state in an optical-fiber coated with Langmuir-Blodgett films, J. Appl.Phys. 78, pp 1367-1369 (1995) 
23 R B Charters, A Kuczynski, S E Staines, R P Tatam and G J Ashwell, In-Line Fibre Optic Channel Dropping Filter using Langmuir-Blodgett Films, Electron.Lett., 30, 594-595, (1994).

24 R B Charters, S E Staines and R P Tatam, In-line fiber-optic components using LangmuirBlodgett, Opt. Lett., 19, 2036-2038 (1994).

25 S.L. Zhao, W.M. Reichert, Modelling of fluorescence emission from cyanine-dye-impregnated langmuir-blodgett-films deposited on the surface of an optical fiber, Thin Solid Films, 200, pp 363-373 (1991)

26 D Flannery, S W James, R P Tatam and G J Ashwell, pH Sensor using Langmuir-Blodgett overlays on polished optical fibre, Opt.Lett., 22 , 567-569, (1997).

27 D Flannery, S W James, R P Tatam and G J Ashwell, Fibre optic chemical sensing using Langmuir-Blodgett overlay waveguides, Appl.Opt. 38 , 7370-7374, 1999.

28 GJ Ashwell, DS Gandolfo, Molecular rectification: dipole reversal in a cationic donor- $(\pi$ bridge)-acceptor dye, J. Mater. Chem. 12, 411-415 (2002).

29 R.H. Selfridge, S.T. Kowel, P. Stroeve, J.Y.S. Lam and B.G. Higgins, Optical fibers coated with non-linear langmuir-blodgett films Thin Solid Films 160, 471-476 (1988)

30 G J Ashwell, W Crossland, P J Martin, P A Thompson, A T Hewson, S Marsden A 2-legged spacer molecule for alternate layer $\mathrm{lb}$ film deposition with optically nonlinear dyes Mat. Res. Soc. Proc. 247, 787, 1992.

31 S S Johal, S W James, R P Tatam and G J Ashwell, SHG in Langmuir-Blodgett waveguide overlays on single mode optical fibre, Opt.Lett., 24, 1194-1196 (1999).

32 S.S. Johal, S.E. Staines, S.W. James, R.P. Tatam and G.J. Ashwell, A technique for depositing non-centrosymmetric Langmuir-Blodgett films onto optical fibres, Meas.Sci.Technol. 10, N60-N62 (1999).

33 C. Bosshard, M. Flörsheimer, M. Küpfer and P. Günter, Opt. Commun., 85, 247, 1991. 
34 S.W. James and R.P. Tatam, Optical Fibre Long Period Grating Sensors: Characteristics and Application Meas.Sci.Technol. 14, R49-R61, (2003).

35 S Khaliq, S W James and R P Tatam, Enhanced sensitivity fibre optic long period grating temperature sensor, Meas. Sci. Technol. 13 792-5 (2002)

36 V. Bhatia, Applications of long-period gratings to single and multi-parameter sensing, Opt. Express, 4, 457-466 (1999).

37 R. Falciai, A.G. Mignani and A. Vannini, Long period gratings as solution concentration sensors Sens. Actuators B-Chemical 74, 74-77 (2001)

38 D.M. Costantini, C.A.P. Muller, S.A. Vasiliev, H.G. Limberger, and R.P. Salathe Tunable loss filter based on metal-coated long-period fiber grating IEEE Photonics Technol. Lett. 11, $1458-1460$ (1999)

39 J.N. Jang, S.Y. Kim, S.W. Kim, M.S. Kim, Temperature insensitive long-period fibre gratings Electron. Lett. 35, 2134-2136 (1999)

40 Z. Zhang, M. Shiloach, S. Pilevar, C.C. Davis, J. Sirkis and Bentley, Evanescent wave long period fiber Bragg grating as an immobilized antibody biosensor Anal. Chem. 72, 2895-2900 (2000).

41 N Rees, S W James, R P Tatam, and G J Ashwell., Optical fiber long period gratings with Langmuir-Blodgett thin film overlays, Opt.Lett., 9, 686-688 (2002).

42 K. Skjonnemand, $\mathrm{PhD}$ thesis, Cranfield University (2000).

43 I Del Villar, MAchaerandio, I.R. Matias, F.J. Arregui, Deposition of overlays by electrostatic self-assembly in long-period fiber gratings, Opt.Lett. 30 720- 722 (2005).

44 Z.Y. Wang, J.R. Heflin, R.H. Stolen, S. Ramachandran, Highly sensitive optical response of optical fiber long period gratings to nanometer-thick ionic self-assembled multilayers Appl.Phys.Lett. 86, 223104 (2005) 
45 A.K. Ghansk, K, Thyagarajan and M.R. Shenoy, Numerical-analysis of planar optical waveguides using matrix approach IEEE J.Lightwave Technol. LT-5, 660-667 (1987).

46 D. Gloge, Weakly guiding fibers Appl.Opt. 10, 2252 (1971)

47 I. Del Villar, I. R. Matias, F.J. Arregui, M. Achaerandio Nanodeposition of Materials With Complex Refractive Index in Long-Period Fiber Gratings, J. Lightwave Technol. 23, 4192$4199(2005)$

48 A Cusano, A Iadicicco, P Pilla, L Contessa, S Campopiano, A Cutolo and M Giordano, Cladding mode reorganization in high-refractive-index-coated long-period gratings: effects on the refractive-index sensitivity. Opt.Lett. 30, 2536-2538 (2005)

49 I. Del Villar, PhD Thesis, Universida Publica de Navarra, (2006)

50 Z Wang, J.R. Helfin, R.H. Stoeln and S Ramachandran, Analysis of the optical response of long period fibre gratings to nm-thick thin-film coatings, Optics Express, 13, 2808-2813, (2005).

52 I Ishaq, S W James, G J Ashwell, R P Tatam, Modification of the refractive index response of long period gratings using thin film overlays, Sensors and Actuators B: Chemical, 107, pp738-741 (2005)

53 I Del Villar, IR Matias, FJ Arregui, Enhancement of sensitivity in long-period fiber gratings with deposition of low-refractive-index materials Opt. Lett. 30, 2363-2365 (2005)

54 A. Cusano, P. Pilla, L. Contessa, A. Iadiccio, S. Campopiano, A. Cutolo, M. Giordano and G. Guerra, High sensiitivity optical chemosesnor based on coated long-period gratings for subppm chmaical detection in water. Appl.Phys.Lett. 87, 234105 (2005).

55 B H Lee and J Nishii Bending sensitivity of in-series long period fibre gratings Opt. Lett. 23, $1624-1626(1998)$

56 S W James, I Ishaq, G J Ashwell and R P Tatam, Cascaded long period gratings with nanostructured coatings, Opt. Lett., 30, 2197-2199 (2005) 
57 F.J. Grunthaner, A.J Ricco, M.A Butler, A.L. Lane, C.P. Mckay, A.P. Zent, R.C. Quinn, B. Murray, H.P. Klein, G.Y. Levin, R.W Terhune, M.L. Homer, A. Ksendzov, P. Niedermann , Investigating the surface-chemistry of mars, Anal.Chem. 67 A605-A610 (1995).

58 D.A. Jackson, Recent progress in monomode fibre-optic sensors Meas.Sci.Technol. 5, 621638 (1994).

59 E.Udd Fiber Optic Smart Structures Wiley Interscience NY (1994).

60 N D Rees, S W James, S E Staines, G J Ashwell and R P Tatam, Submicrometer fiber optic Fabry-Perot interferometer formed by the use of the Langmuir-Blodgett technique Opt. Lett., 26 , 1840-1842, (2001).

61 F J Arregui, I R Matias, Y J Liu, K M Lenahan, R O Claus Optical fiber nanometer-scale Fabry-Perot interferometer formed by the ionic self-assembly monolayer process, Opt. Lett. 24 596-598 (1999)

62 F.J. Arregui, K..L Cooper, Y.J. Liu, I.R. Matias, R.O. Claus Optical fiber humidity sensor with a fast response time using the ionic self-assembly method, IEICE Transactions On Electronics E83C, 360-365 (2000)

63 F. J. Arregui, R.O. Claus, K. L. Cooper, C. Fernández-Valdivielso, and I. R. Matías Optical Fiber Gas Sensor Based on Self-Assembled Gratings, , J.Lightwave.Technol. 19, 1932-1936 $(2001)$

64 C Bariain, I.R .Matias, C. Fernandez-Valdivielso, F.J. Arregui, M.L. Rodriguez-Mendez, J.A. de Saja Optical fiber sensor based on lutetium bisphthalocyanine for the detection of gases using standard telecommunication wavelengths Sens.Act. B-Chemical 93 153-158 (2003).

65 D. Galbarra, F.J. Arregui, I.R. Matias, R.O. Claus, Ammonia optical fiber sensor based on self-assembled zirconia thin films Smart Mat.Struct. 14 739-744 (2005) 
66 I Del Villar, IR Matias, FJ Arregui, J Echeverria, RO Claus, Strategies for fabrication of hydrogen peroxide sensors based on electrostatic self-assembly (ESA) method, Sens.Act. BChemical 108 751-757 (2005)

67 I. Del Villar, I.R. Matias, F.J. Arregui, R.O. Claus, ESA-based in-fiber nanocavity for hydrogen-peroxide detection IEEE Trans. Nanotechnology 4 187-1932005.

68 FJ Arregui, IR Matias, KL Cooper, and R.O. Claus, Fabrication of microgratings on the ends of standard optical fibers by the electrostatic self-assembly monolayer process, Opt.Lett. 26 $131-1332001$. 


\section{Figure Captions}

Figure 1. Idealised pressure-area isotherm for a Langmuir film formed on a sub-phase of pure water.

Figure 2. $\quad$ Principles of LB deposition. (a) Y-type, centrosymmetric deposition. (b) Z-type, noncentrosymmetric deposition. Organic molecule with hyrodophilic $(\bullet)$ and hydrophobic ends.

Figure 3. Schematic illustration of the deposition of a multilayer film by consecutive adsorption of anionic and cationic polyelectrolytes [from [11]].

Figure 4. Polished fibre configurations. (a) Single mode optical fibre fixed into a groove on a 250 $\mathrm{mm}$ radius of curvature, and polished using a rotary polishing machine. (b) Annularly polished optical fibre.

Figure 5. Transmission spectra of a side polished optical fibre with overlay waveguide of $\omega$ tricosenoic acid deposited via the LB technique. The curves from left to right show the spectra for 112, 114, 116 and 118 layers respectively [from 23].

Figure 6. (a) Thermal response of the channel dropping spectrum. The temperature range is (left to right) $42^{\circ} \mathrm{C}$ to $9.7^{\circ} \mathrm{C}$, (b) Thermal tuning response of figure 5 (a) [from 24].

Figure 7. Schematic illustration of the experimental system to capture the fluorescence from an LB film deposited on an optical fibre. The core sizes of fibers I and 2 are $200 \mathrm{pm}$ and $600 \mathrm{pm}$ respectively. $\mathrm{fl}$ and $\mathrm{f} 2$ are the $488 \mathrm{~nm}$ passing and rejection filters respectively [from[25]].

Figure 8. (a) Molecular structure of E-N-docosyl-4-[2-(4-oxyphenyl) ethenyl] pyridinium inner salt (b) Absorption spectra of E-N-docosyl-4-[2-(4-oxyphenyl) ethenyl] pyridinium inner salt before and after exposure to $\mathrm{HCl}$ vapour [from 27].

Figure 9. Schematic diagram of the polished fibre with the LB thin film overlay. 
Figure 10 (a) Normalised, TM transmission of sensor: from left to right $11.52 \mathrm{pH}, 11.99 \mathrm{pH}$, $12.31 \mathrm{pH}, 12.67 \mathrm{pH}$ and $13.16 \mathrm{pH}$. (b) Plot of coupling wavelength versus solution $\mathrm{pH}$ [from 27].

Figure 11. (a) Molecular structure of bromine Merocyanine. (b) Absorption spectra of 50 layers of the merocyanine dye, before and after exposure to $\mathrm{HCl}$ vapour [from 27 ]. ${ }^{20}$.

Figure 12. (a) Normalised TM transmission of sensor, in solutions of, from left to right, 11.60, $11.83,12.09,12.39,12.64,12.80,13.02$ and $13.23 \mathrm{pH}$. (b) Coupling wavelength versus solution $\mathrm{pH}$ showing nonlinear response of the sensor [from 27]..

Figure 13. Normalised spectral response of overlay device with a drop in transmission of $\sim 13 \mathrm{~dB}$ [from 32].

Figure 14. Schematic of the Cerenkov-type phase matching configuration.

Figure 15. Graph of SHG intensity as a function of fibre transmission. Constant pump power coupled into the fibre. Thermal tuning of $\lambda_{m}$ causes the power coupled to the LB overlay to change [from 32]..

Figure 16. Graph of SHG intensity as a function of fiber coupled power at $\mathrm{T}=21.4{ }^{\circ} \mathrm{C}[$ from 32$]$.

Figure 17. Transmission spectrum of a long period grating of length $40 \mathrm{~mm}$ and period $400 \mu \mathrm{m}$, written into photosensitive optical fibre with a cut-off wavelength of $644 \mathrm{~nm}$ [from 35].

Figure 18. The spectral response of an LPG of length $40 \mathrm{~mm}$, period $400 \mu \mathrm{m}$, to submersion in external media of different refractive indices [from 35].

Figure 19. (a) Experimental determined central wavelengths of the attenuation bands plotted as a function of the thickness of the overlay film. - Attenuation band corresponding to coupling to the $5^{\text {th }}$ cladding mode, $\mathbf{\square}$ attenuation band corresponding to coupling to the $6^{\text {th }}$ cladding mode. (b) corresponding wavelength shifts, labeling the attenuation bands from the shorter to longer wavelengths [from 41].

Figure 20. (a) Theoretically predicted wavelength of the central wavelengths of the attenuation bands plotted as a function of the thickness of an overlay film of refractive index 1.57. 
(b) corresponding wavelength shifts, labeling the attenuation bands from the shorter to longer wavelengths. $\mathrm{x}$ attenuation band corresponding to coupling to the 2 nd cladding mode attenuation band corresponding to coupling to the $3^{\text {rd }}$ cladding mode attenuation band corresponding to coupling to the $4^{\text {th }}$ cladding mode $\bullet$ attenuation band corresponding to coupling to the $5^{\text {th }}$ cladding mode, a attenuation band corresponding to coupling to the $6^{\text {th }}$ cladding mode.

Figure 21. Effective refractive indices of the cladding modes, calculated using the LP approximation, as a function of overlay thickness, illustrating the reorganisation of the cladding modes during the transition to guidance by the overlay. [from 43].

Figure 22. Theoretical prediction of the influence on the refractive index of the thin film overlay on the sensitivity of the LPG transmission spectrum to overlay thickness. $\boldsymbol{\Delta}$ refractive index $2, \bullet$ refractive index $1.7, \square$ refractive index 1.57 .

Figure 23. Theoretical prediction of the LPG transmission spectrum on changes in the refractive index of a film of thickness $150 \mathrm{~nm}$.

Figure 24. A schematic diagram of concatenated LPGs, illustrating the beam paths that form the Mach-Zehnder interferometer.

Figure 25. Transmission spectrum of concatenated LPGs, of length $40 \mathrm{~mm}$, period $400 \mu \mathrm{m}$ and separation 20mm. [from 56]

Figure 26. Wavelength shifts of the minima of the fringes in the $5^{\text {th }}$ attenuation band of concatenated LPGs of length $5 \mathrm{~mm}$, period $400 \mu \mathrm{m}$, separated by $20 \mathrm{~mm}$, as a function of the thickness of an LB film deposited on the length of fibre separating the LPGs. [from 56]

Figure 27. The Langmuir-Blodgett technique deployed on the distal end of an optical fibre.

Figure 28. (a) Experimental setup, where SLD is a superluminescent diode, OPM 1 and OPM 2 are the inputs of a dual channel optical power meter, $\mathrm{C}$ is the $3 \mathrm{~dB}$ directional fibre coupler 
and the cavity is fabricated using the LB technique. (b) The cavity formed at the end of the fibre, and the designated refractive indices.

Figure 29. Plot of reflected optical power against number of bilayers deposited. [from $\underline{60}$ ] 


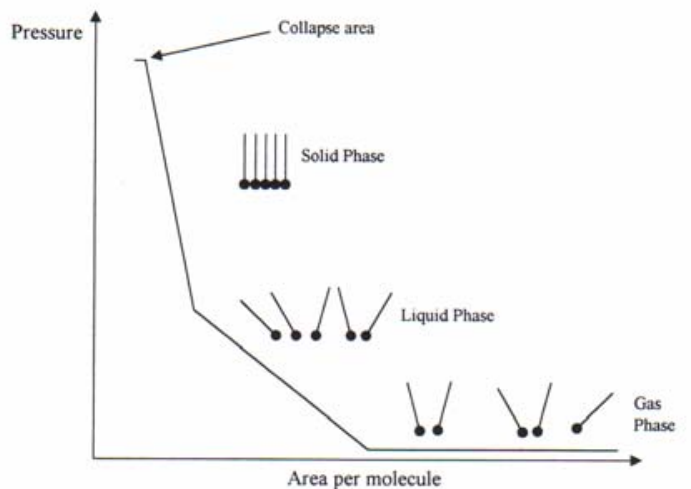

Figure 1. 


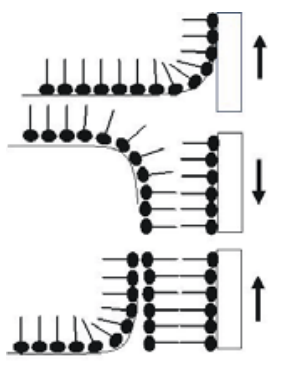

(a)

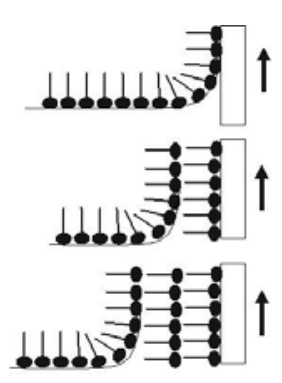

(b)

Figure 2. 

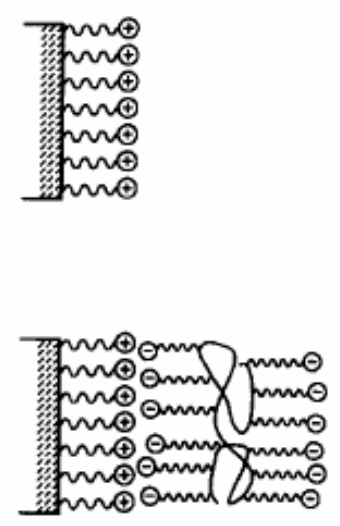

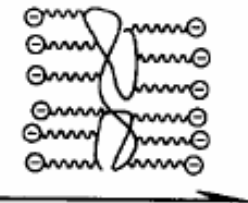

A

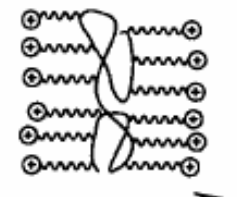

B

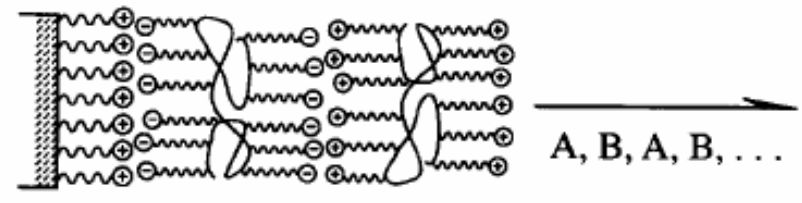

Figure 3 


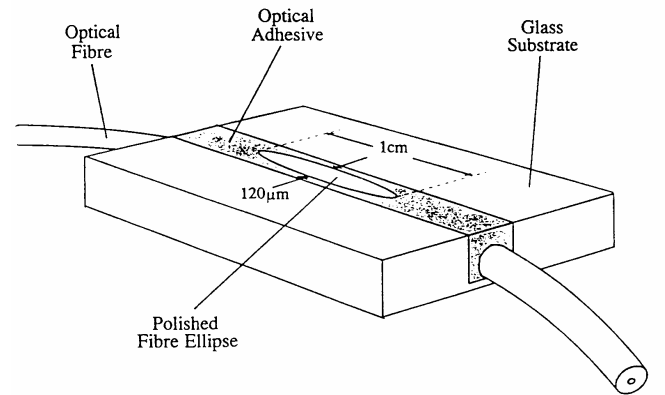

(a)

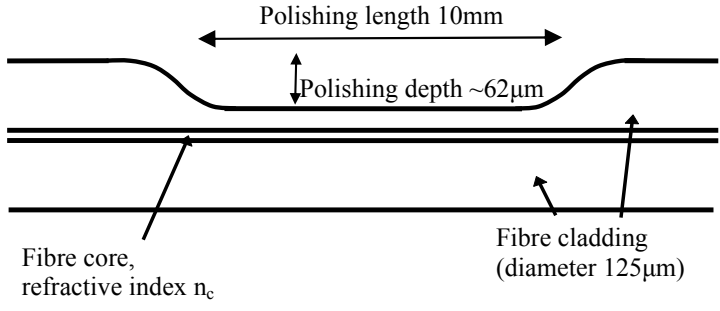

(b)

Figure 4. 


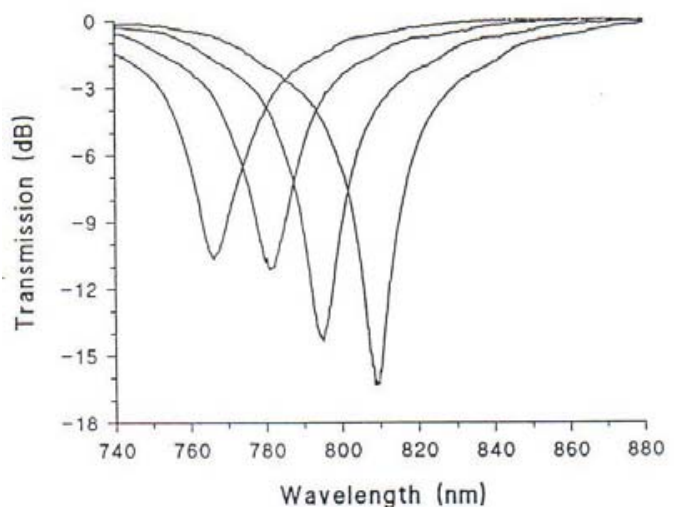

Figure 5. 


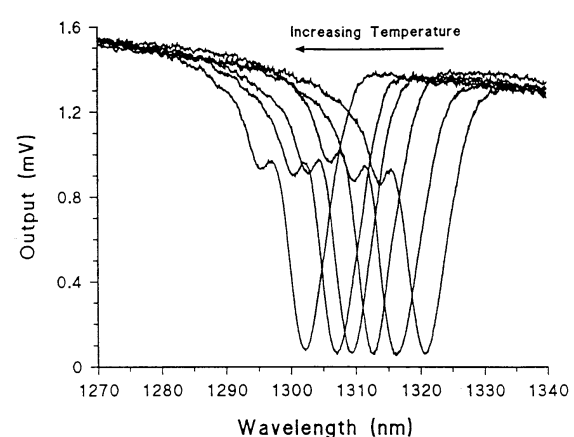

(a)

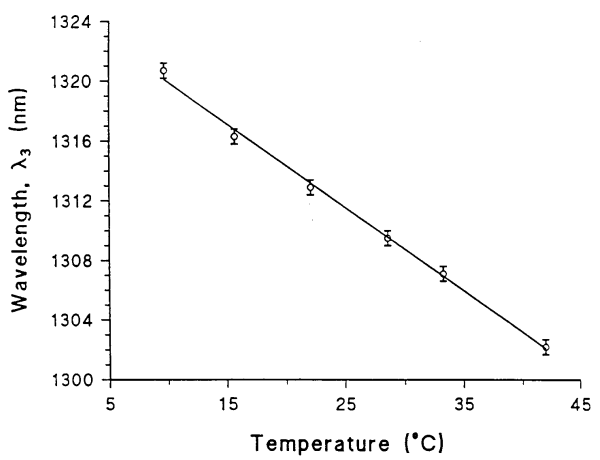

(b)

Figure 6 


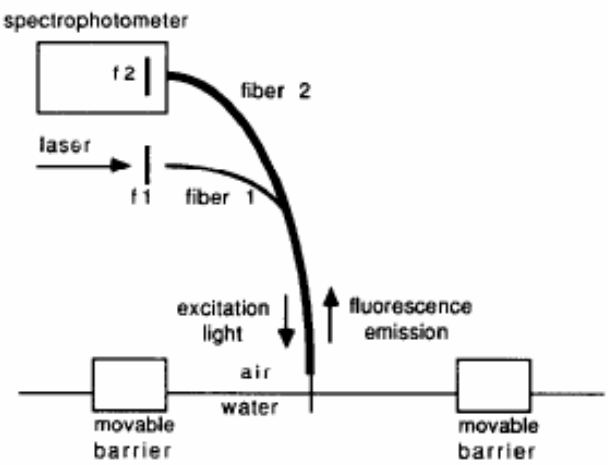

Figure 7 


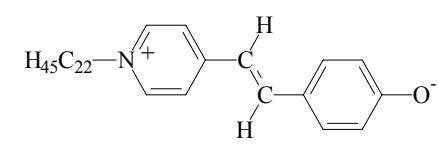

Figure 8(a).

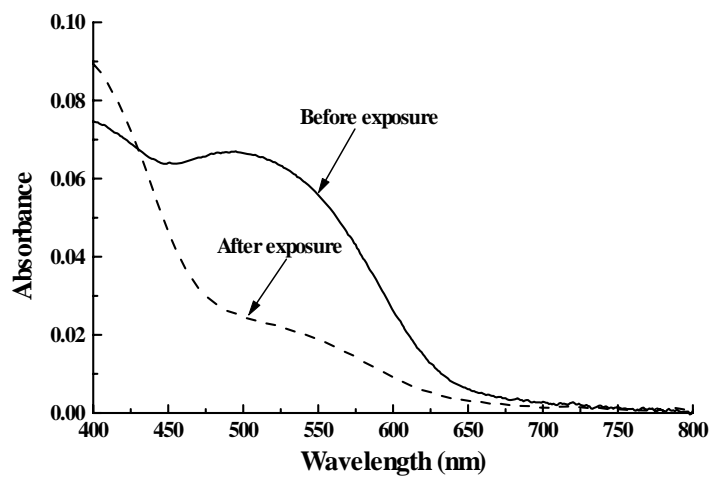

Figure 8(b). 
Langmuir-Blodgett film,

refractive index $n_{0}$

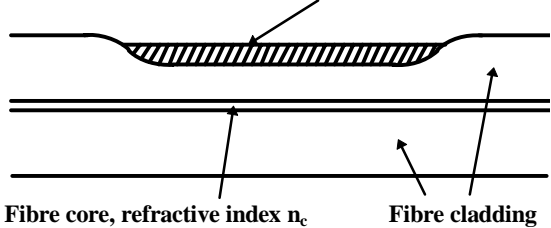

Figure 9. 


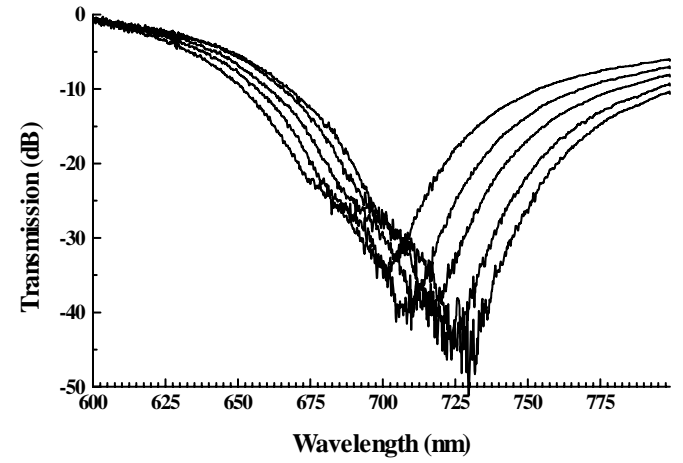

(a)

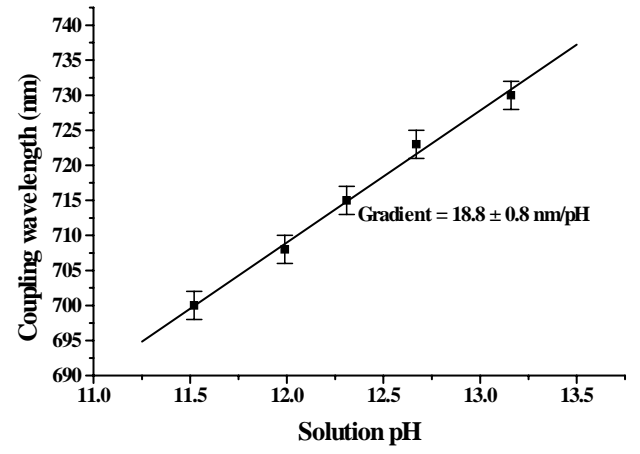

(b)

Figure 10 


\begin{tabular}{|l|l|}
\hline \\
\hline Figure 11
\end{tabular} (a)




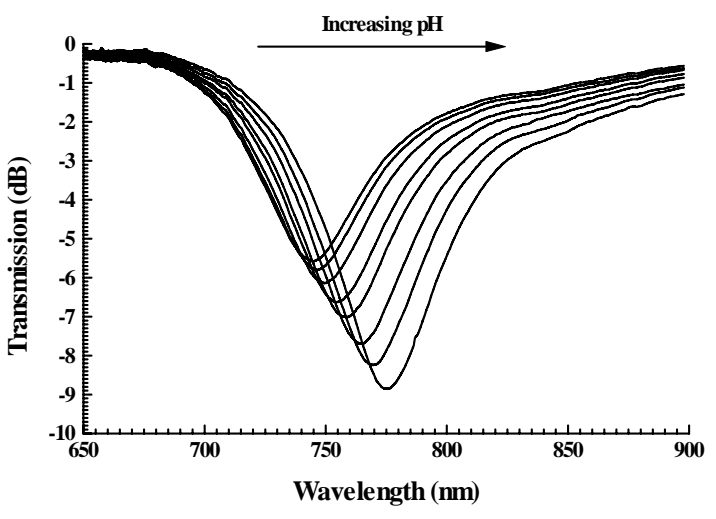

(a)

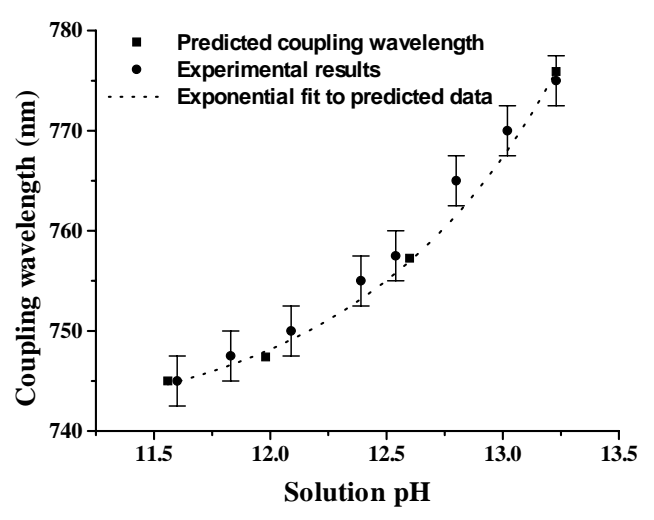

(b)

Figure 12 


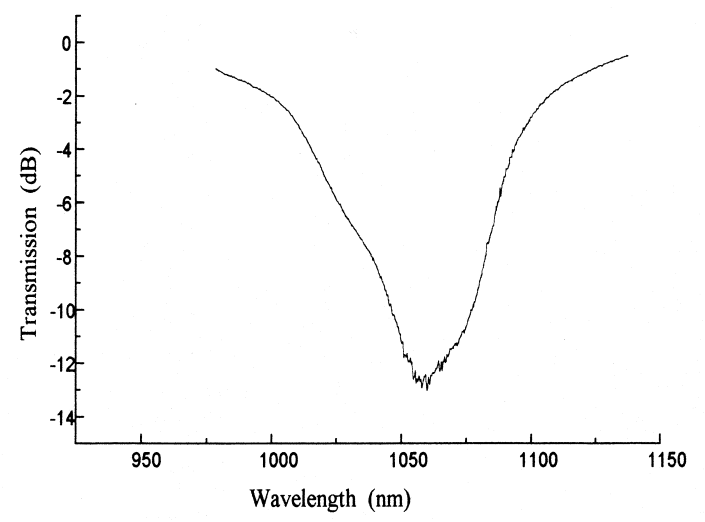

Figure 13 
Fundamental wave

Second harmonic radiation

Substrate

Figure 14 


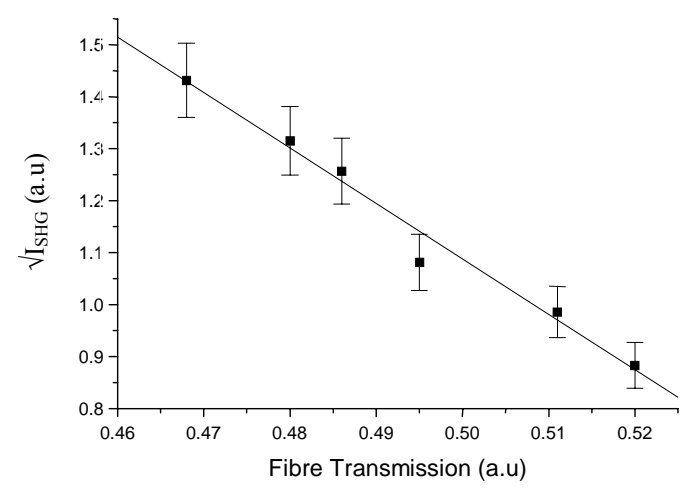

Figure 15 


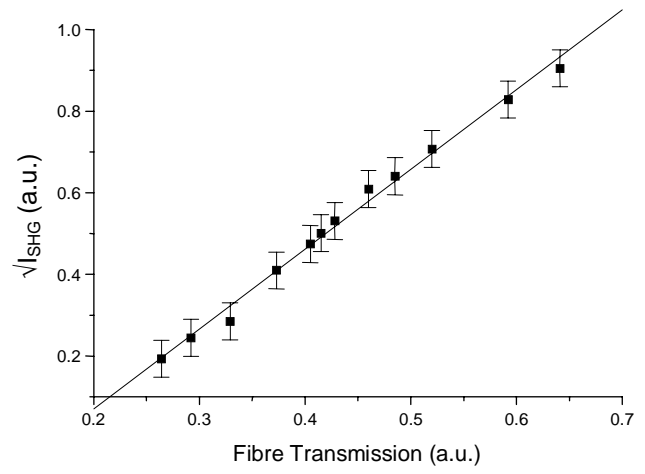

Figure 16 


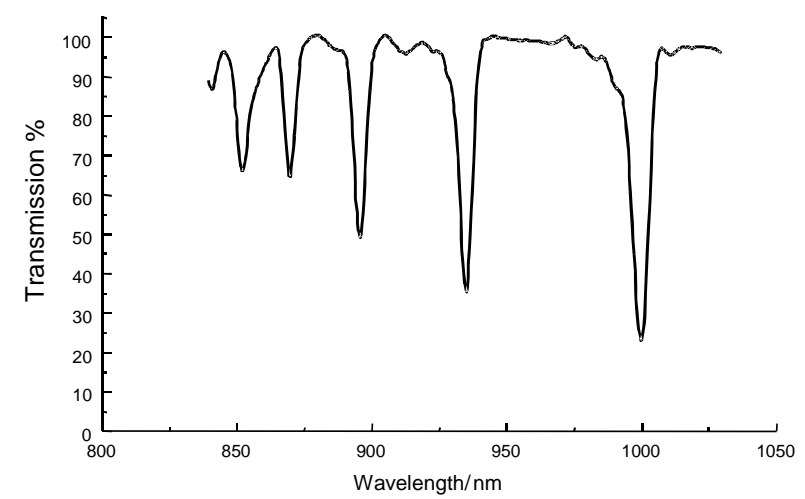

Figure 17 


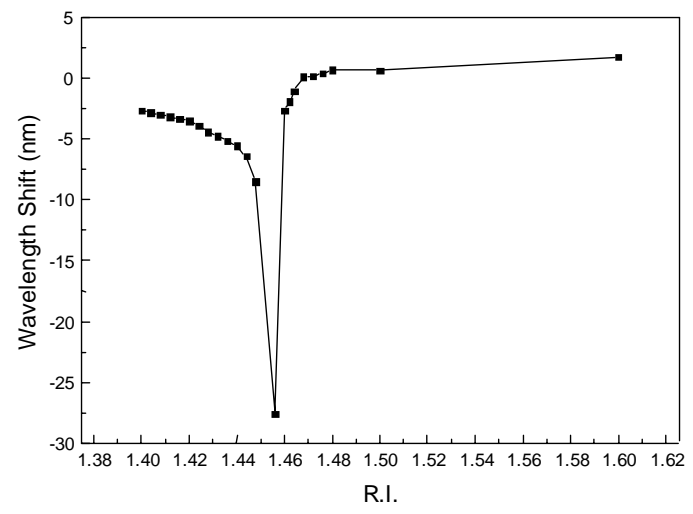

Figure 18 


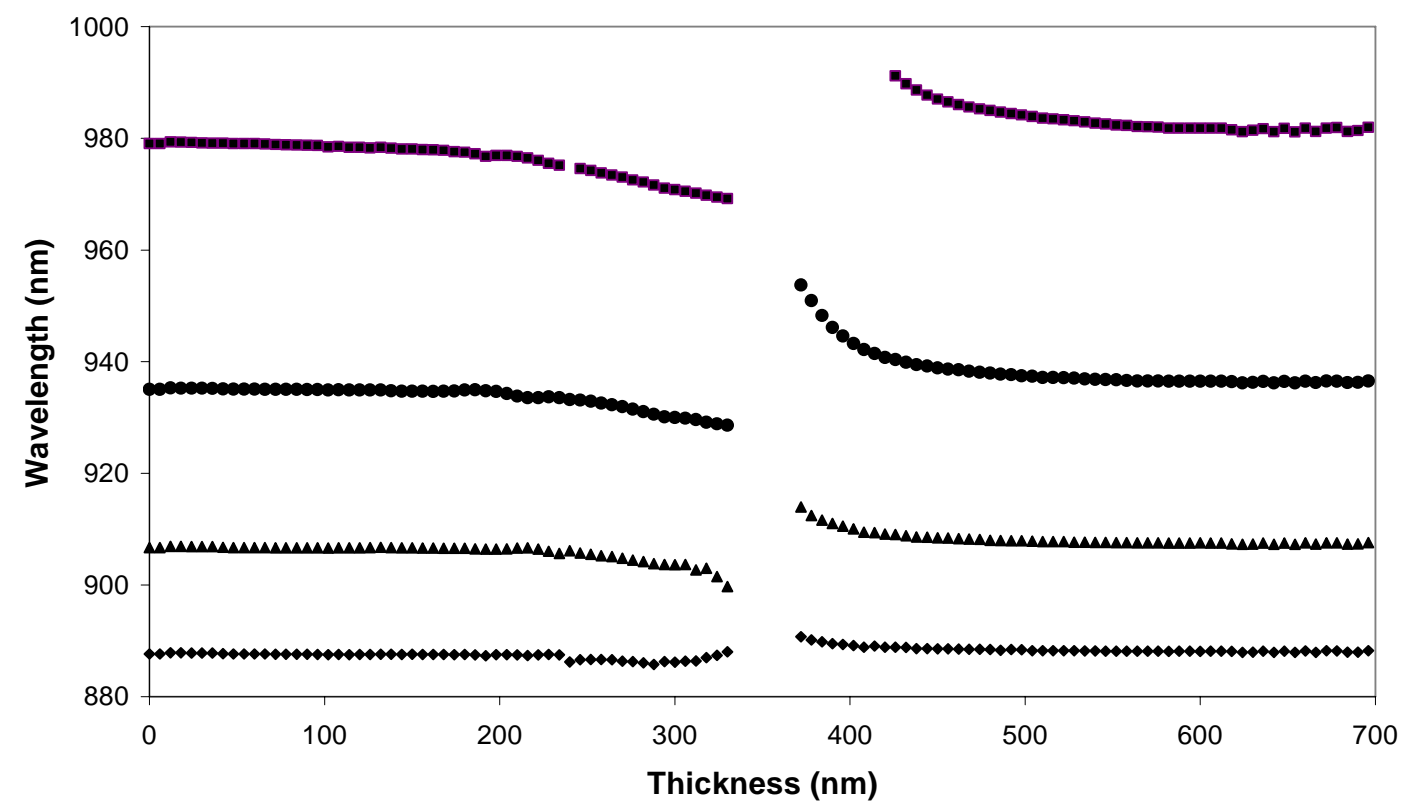

(a)

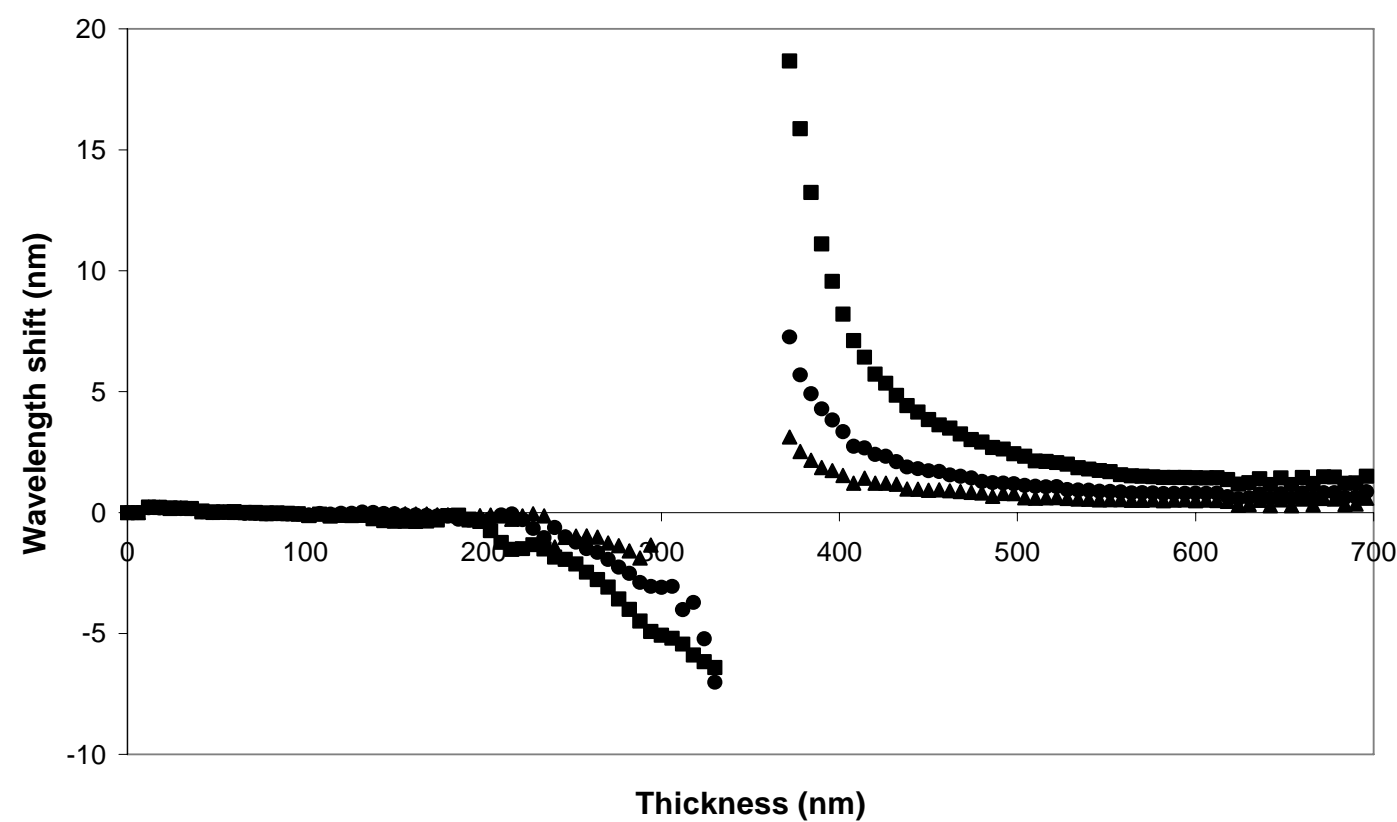

(b)

Figure 19. 


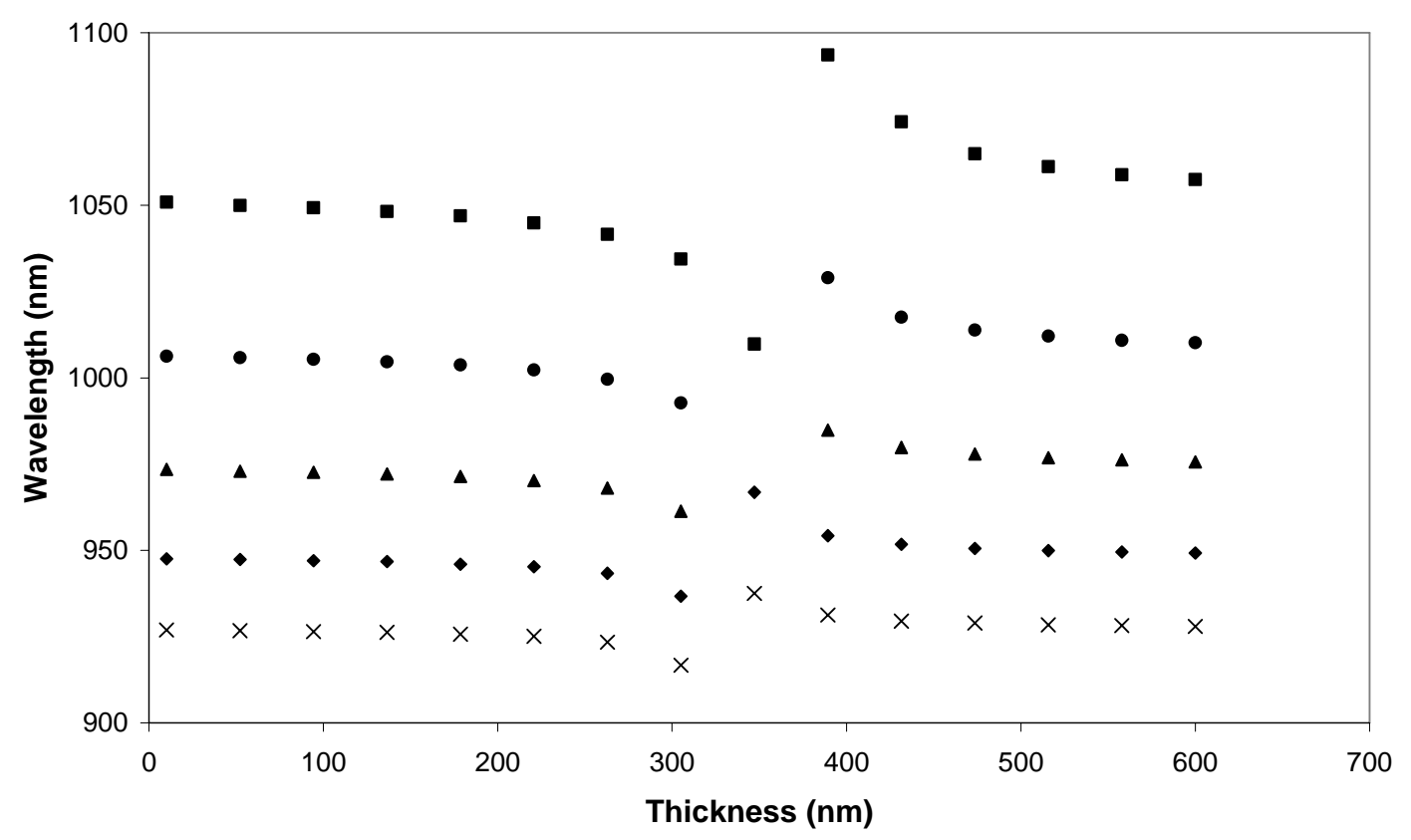

Figure 20 (a)

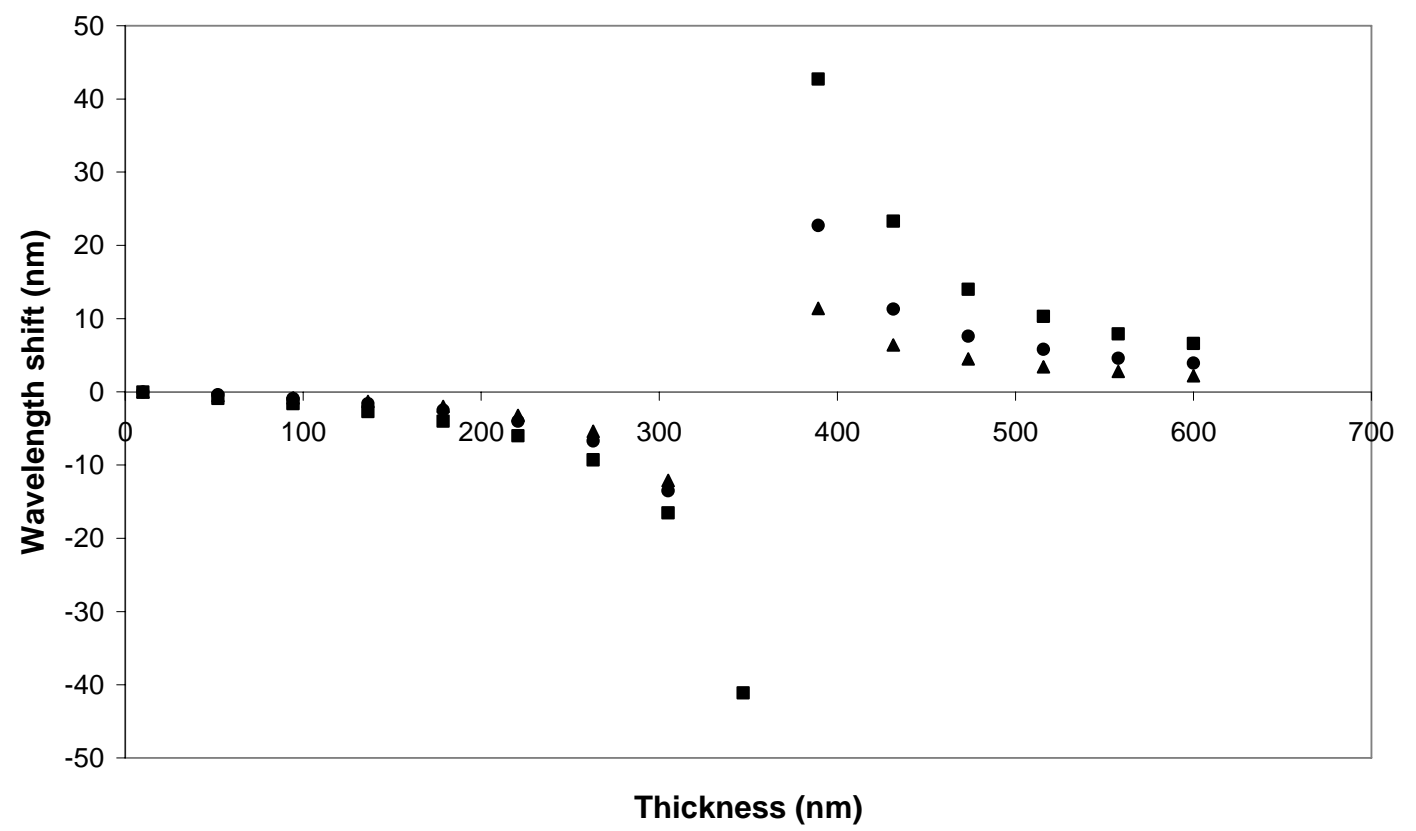

Figure 20 (b) 


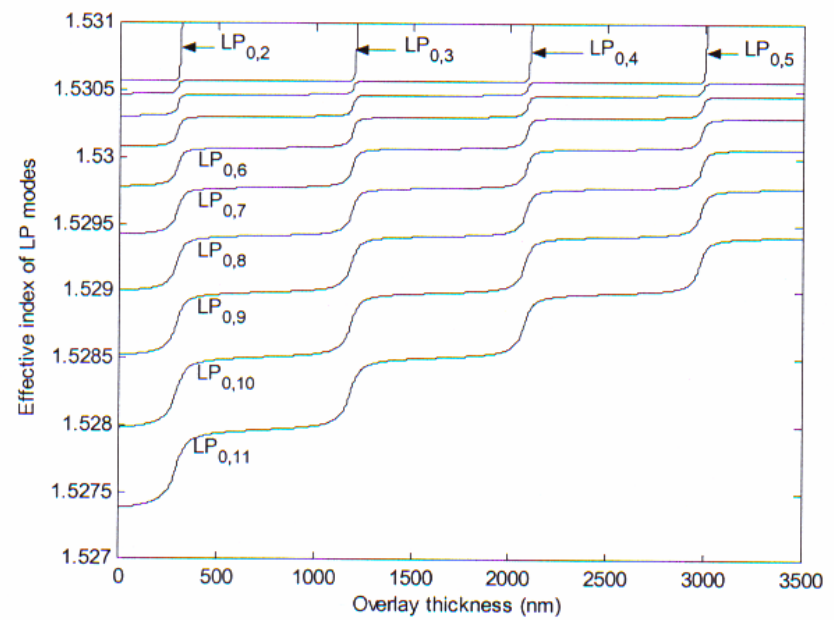

Figure 21. 


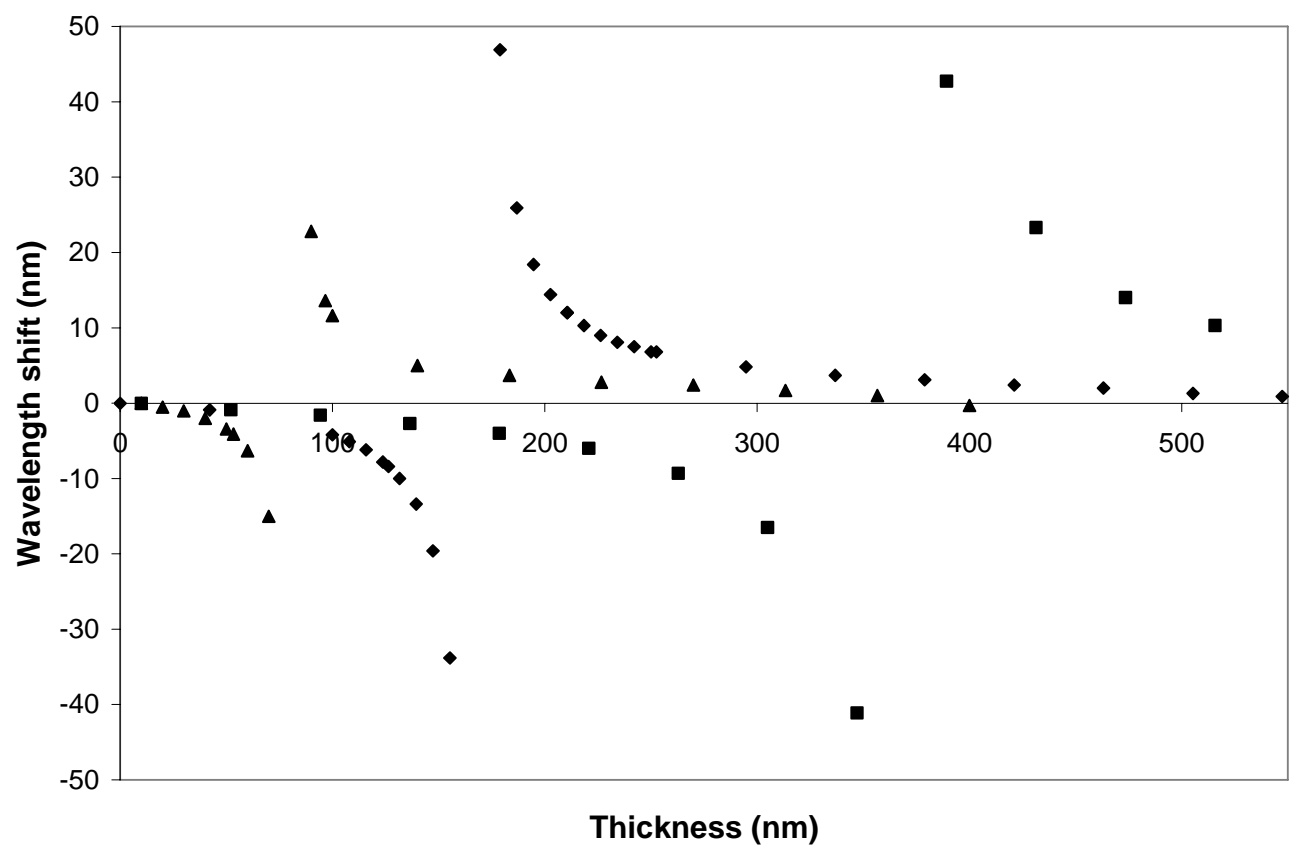

Figure 22 


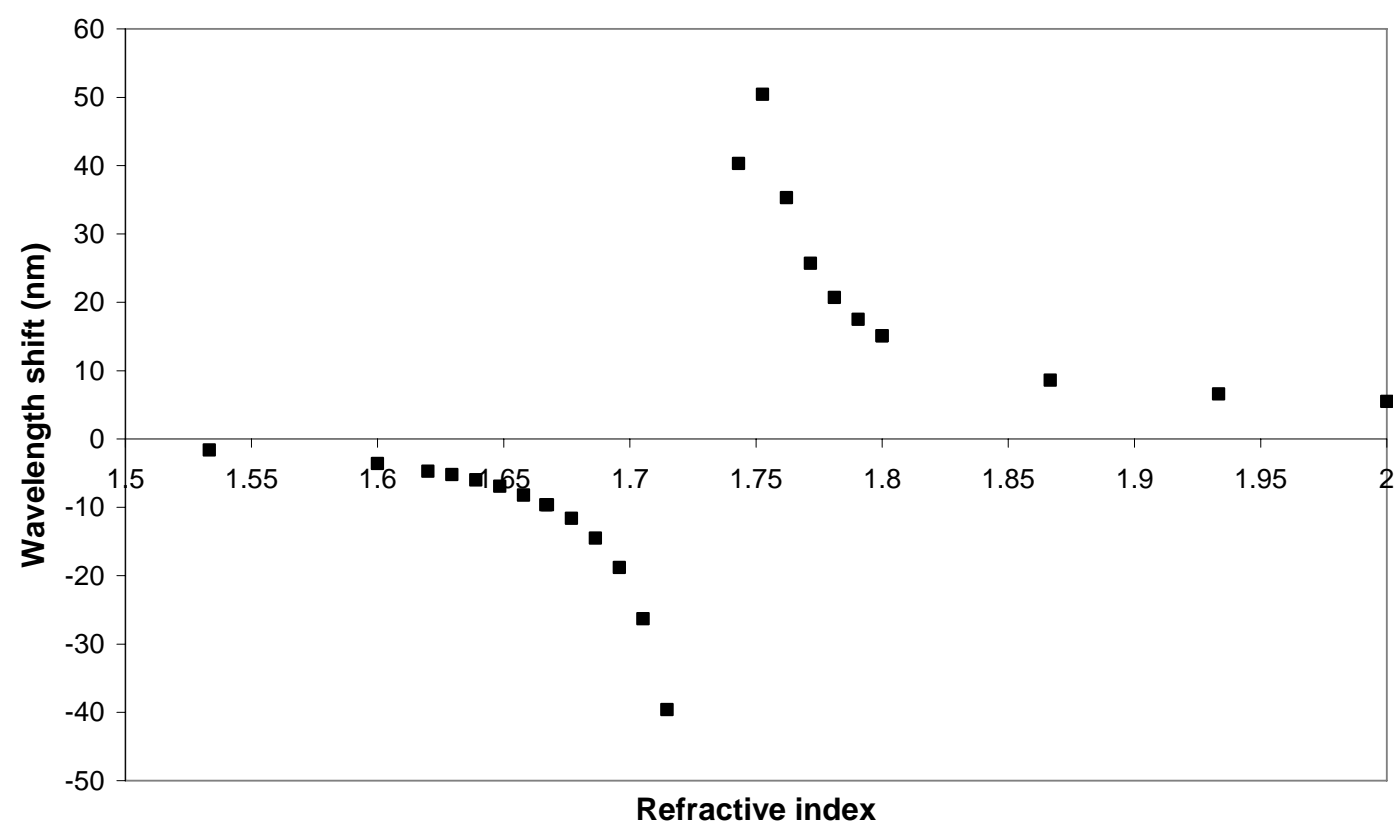

Figure 22 


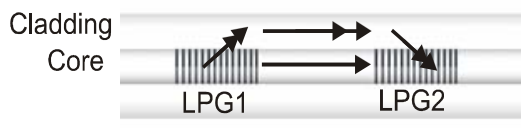

Figure 23 


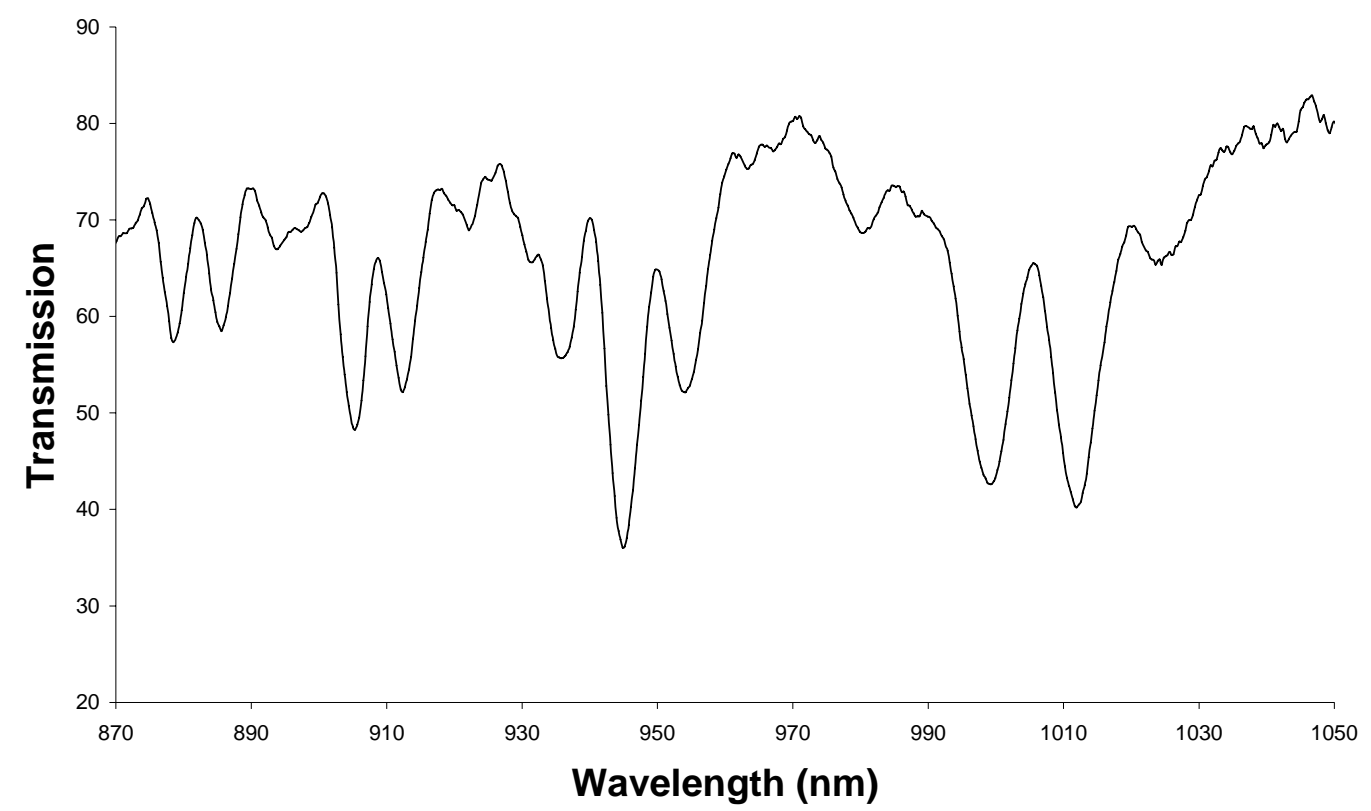

Figure 24 


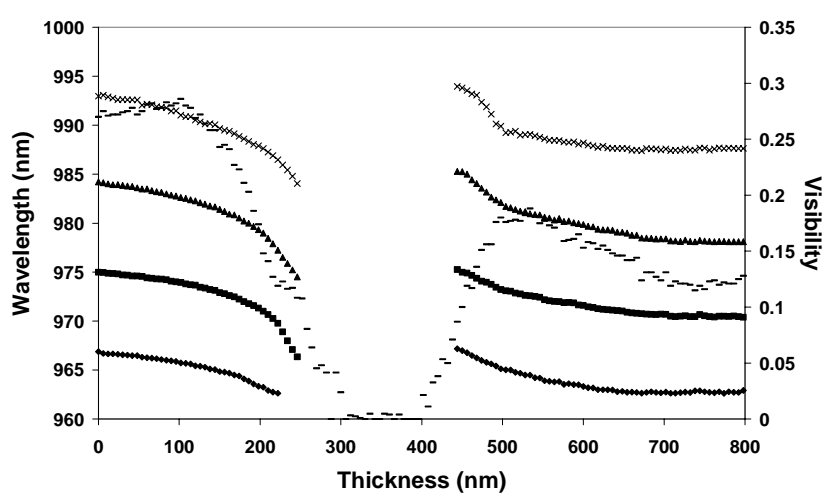

Figure 25 


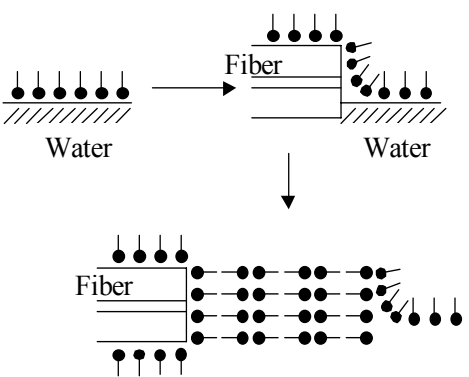

Figure 26. 
(a)

SLD

OPM 2

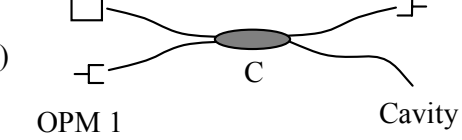

(b)

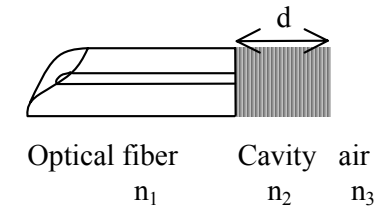

Figure 27 


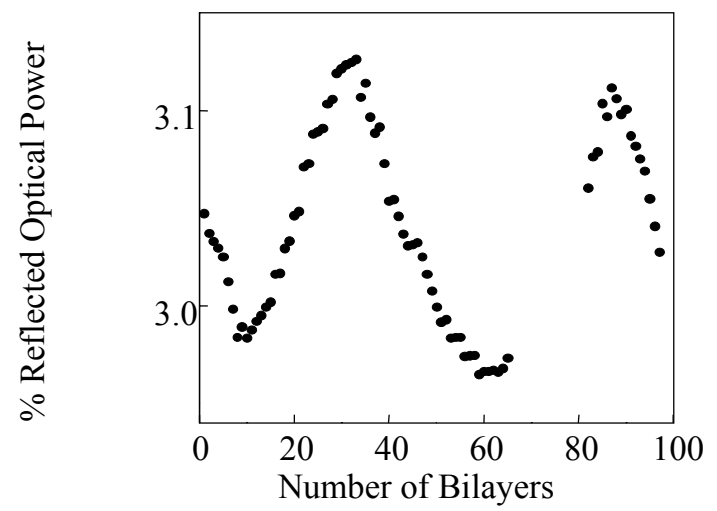

Figure 28 